\title{
Diagnosis of Alzheimer's Disease from EEG Signals: Where Are We Standing?
}

\author{
Justin Dauwels ${ }^{* \dagger}$, François Vialatte ${ }^{+}$, and Andrzej Cichocki ${ }^{+}$ \\ * Laboratory for Information and Decision Systems \\ Massachusetts Institute of Technology, Cambridge, MA \\ $\dagger$ Amari Research Unit \\ RIKEN Brain Science Institute, Saitama, Japan \\ ${ }^{+}$Laboratory for Advanced Brain Signal Processing \\ RIKEN Brain Science Institute, Saitama, Japan
}

\begin{abstract}
This paper reviews recent progress in the diagnosis of Alzheimer's disease (AD) from electroencephalograms (EEG). Three major effects of AD on EEG have been observed: slowing of the EEG, reduced complexity of the EEG signals, and perturbations in EEG synchrony. In recent years, a variety of sophisticated computational approaches has been proposed to detect those subtle perturbations in the EEG of AD patients. The paper first describes methods that try to detect slowing of the EEG. Next the paper deals with several measures for EEG complexity, and explains how those measures have been used to study fluctuations in EEG complexity in AD patients. Then various measures of EEG synchrony are considered in the context of AD diagnosis.

Also the issue of EEG pre-processing is briefly addressed. Before one can analyze EEG, it is necessary to remove artifacts due to for example head and eye movement or interference from electronic equipment. Pre-processing of EEG has in recent years received much attention. In this paper, several state-of-the-art pre-processing techniques are outlined, for example, based on blind source separation and other non-linear filtering paradigms.

In addition, the paper outlines opportunities and limitations of computational approaches for diagnosing $\mathrm{AD}$ based on EEG. At last, future challenges and open problems are discussed.
\end{abstract}

Keywords: Alzheimer's disease (AD), mild cognitive impairment (MCI), Electroencephalography (EEG), synchrony, slowing, complexity, differential diagnosis, cognitive decline, medication 


\section{List of Abbreviations}

- AD: Alzheimer's disease

- AERP: auditory event-related potential (ERP)

- ARMA model: autoregressive-moving average model

- BESA: Brain Electric Source Analysis

- BSS: blind source separation

- DTF: directed transfer function

- DTI: diffusion tensor imaging

- ECG: electrocardiogram

- EEG: electroencephalogram

- EMG: electromyogram

- EP: evoked potential

- EOG: electro-oculogram

- ERD: event-related desynchronization

- ERP: event-related potential

- ffDTF: full-frequency directed transfer function (DTF)

- GFS: global field synchrony

- LORETA: low-resolution brain electromagnetic tomography

- MCI: mild cognitive impairment

- MMSE: mini mental state examination

- MRI: magnetic resonance imaging

- PCA: principal component analysis

- PDC: partial directed coherence

- PLI: phase lag index

- PMCI: progressive mild cognitive impairment (MCI)

- REM sleep: rapid eye movement sleep

- SES: stochastic event synchrony

- SMC: subjective memory complaints

- SMCI: stable mild cognitive impairment (MCI)

- SPECT: single photon emission computed tomography

- SSR: steady-state response

- TMS: transcranial magnetic stimulation 


\section{Introduction}

Alzheimer's disease $(\mathrm{AD})$ is a neuro-degenerative disease, the most common form of dementia, third most expensive disease and sixth leading cause of death in the United States. It affects more than $10 \%$ of Americans over age 65 , nearly $50 \%$ of people older than 85 , and it is estimated that the prevalence of the disease will triple within the next 50 years $[1,2]$. While no known cure exists for Alzheimer's disease, a number of medications are believed to delay the symptoms (and perhaps causes) of the disease.

The progression of the disease can be categorized in four different stages. The first stage is known as Mild Cognitive Impairment (MCI), and corresponds to a variety of symptoms - most commonly memory loss - which do not significantly alter daily life. Between 6 and $25 \%$ of people affected with MCI progress to AD every year. The next stages of Alzheimer's disease (Mild and Moderate $\mathrm{AD})$ are characterized by increasing cognitive deficits, and decreasing independence, culminating in the patient's complete dependence on caregivers and a complete deterioration of personality (Severe AD) [3].

Diagnosis of MCI and AD is important for several reasons $[4,5,6,7,8]$ :

- A positive diagnostic gives the patient and his family time to inform themselves about the disease, to make life and financial decisions related to the disease, and to plan for the future needs and care of the patients.

- A negative diagnostic may ease anxiety over memory loss associated with aging. It also allows for early treatments of reversible conditions with similar symptoms (such as thyroidal problems, depression, and nutrition or medication problems).

- Current symptoms-delaying medications have a given time frame during which they are effective. Early diagnosis of AD helps ensure prescription of these medications when they are most useful.

- Early diagnosis of AD also allows prompt treatment of psychiatric symptoms such as depression or psychosis, and as such reduces the personal and societal costs of the disease.

- As research progresses, preventive therapies may be developed. Early diagnosis raises the chance of treating the disease at a nascent stage, before the patient suffers permanent brain damage.

- Finally, as institutionalization accounts for a large part of health care costs incurred because of $\mathrm{AD}$, by preserving patients' independence longer and preparing families for the needs of AD patients, timely diagnosis further decreases the societal cost of the disease.

Medical diagnosis of Alzheimer's disease is hard, and symptoms are often dismissed as normal consequences of aging. Diagnosis is usually performed through a combination of extensive testing and eliminations of other possible causes. Psychological tests such as Mini Mental State Examinations (MMSE), blood tests, spinal fluid, neurological examination, and increasingly, imaging techniques are used to help diagnose the disease $[9,10,11,12,13]$.

In the last years, several research groups have started investigating the potential of electroencephalograms (EEGs) for diagnosing AD. Since EEG recording 
systems are inexpensive and (potentially) mobile, EEG may potentially be used as a tool to screen a large population for the risk of $\mathrm{AD}$.

In this paper, we review and assess the progress that has been made in recent years in diagnosing AD from EEG signals. Studies have shown that AD has (at least) three major effects on EEG (see [14] for an in-depth review): slowing of the EEG, enhanced complexity of the EEG signals, and perturbations in EEG synchrony. Those effects, however, are not always easily detectable: there tends to be large variability among AD patients. As a result, none of those phenomena allow at present to reliably diagnose AD at an early stage. Many recent studies have investigated how to improve the sensitivity of EEG for detecting AD. In this paper we review and assess those studies.

The paper is structured as follows. We start by reviewing various methods to preprocess EEG signals (Section 2): before EEG signals can be analyzed, they need to be appropriately filtered and artifacts need to be removed. Next we review several state-of-the-art signal processing methods to detect perturbations in EEG signals caused by AD; we will treat the three major effects of AD on EEG separately: slowing of the EEG (Section 3), enhanced complexity of the EEG signals (Section 4), and perturbations in EEG synchrony (Section 5). We assess the potential and limitation of such computational approaches (Section 6), and address remaining challenges and open problems (Section 7). At the end of the paper, we offer some concluding remarks (Section 8).

This paper is one of the few studies in recent years to provide an overview and critical assessment of various state-of-the-art signal processing methods for diagnosing AD from EEG signals (see [15, 16, 17] for related earlier reviews).

\section{Preprocessing}

Before EEG signals can be analyzed, they need to be appropriately processed, for example, to remove artifacts; this section is devoted to such preprocessing methods. We first explain why preprocessing is necessary, and then we outline the state-of-the-art in EEG preprocessing. At the end of this section, we briefly address EEG preprocessing in the context of diagnosing AD.

\subsection{Need for Preprocessing}

EEG recordings typically not only contain electrical signals from the brain, but also several unwanted signals [18, 19, 20, 21]:

- interference from electronic equipment, as for example the 50 or $60 \mathrm{~Hz}$ power supply signals,

- electromyographic (EMG) signals evoked by muscular activity,

- ocular artifacts, due to eye movement or blinking.

Those unwanted components may bias the analysis of the EEG, and may lead to wrong conclusions [21, 22]. Consequently, artifact rejection is an important issue in biomedical [23] and clinical [24] applications of EEG. 
It is often believed that EMG activity is confined to high frequencies (above 20 or $40 \mathrm{~Hz}$ ); therefore, one often applies a low-pass filter to the EEG, with the aim of removing EMG and other artifacts. Several studies have shown, however, that the frequency range of EMG is in fact much wider than commonly believed [25, 26, 27, 24]:

- EMG from skeletal muscles, recorded from the skin, has a frequency distribution from 0 to more than $200 \mathrm{~Hz}$; this EMG signal contains several distinct components: a delta range component $(0-5 \mathrm{~Hz})$, probably associated with common drive to the motor units; an alpha component (about $10 \mathrm{~Hz}$ ), believed to be associated with motor unit firing and physiological tremor; a beta component (20-30 Hz), referred to as EMG beta rhythm; a gamma component $(35-60 \mathrm{~Hz})$, commonly referred to as Piper rhythm.

- EMG from facial muscles also shows a broad frequency distribution, with two peaks corresponding to the EMG beta and Piper rhythms.

Interestingly, muscle artifacts during resting EEG conditions seem to be mostly generated by temporalis and frontalis muscles [28, 29]. Therefore, EMG activity can usually be recognized by its peripheral location on EEG scalp topographs [24].

A related issue is "blurring" or "volume conduction", i.e., the spreading of cortical sources as their voltage propagates to the scalp. This spreading can result in spurious coupling between electrodes, due to a common source influencing two electrodes and producing apparently synchronous activity. As a consequence, it is difficult to relate perturbations in EEG synchrony to physiological phenomena. One option is to try to infer the electrical activity inside the brain; in particular, one commonly assumes that the EEG is generated by a limited number of sources ("source model"), and one tries to localized those sources. Various inverse methods have been developed for this purpose. However, all of those methods have their own drawbacks, and volume conduction remains hard to handle. We will return to this issue in Section 5.3.

\subsection{Preprocessing Methods}

We describe here various preprocessing techniques to remove unwanted signals from EEG.

\subsubsection{Filtering}

The spurious 50 or $60 \mathrm{~Hz}$ power supply signals are typically removed by a bandstop filter, which is a filter that passes most frequencies unaltered, but attenuates those in a specific range (e.g., at 50 or $60 \mathrm{~Hz}$ ) to very low levels. However, other artifacts such as EMG signals and ocular artifacts typically affect a large frequency band and their spectrum may vary over time. Therefore, band-stop filters are usually not effective to eliminate such artifacts.

One is often interested in specific frequency bands in the EEG, such as $4-8 \mathrm{~Hz}$ (theta), $8-10 \mathrm{~Hz}$ (alpha 1), 10-12 Hz (alpha 2), 12-30Hz (beta), and $30-$ $100 \mathrm{~Hz}$ (gamma) [30]. Such frequency bands are usually extracted by a bandpass 
filter, which is a filter that passes frequencies within a certain range and rejects (attenuates) frequencies outside that range.

\subsubsection{Artifact Suppression}

To reduce artifacts, one may simply ask the subjects to suppress eye movement or blinking and to keep still. However, this approach is problematic: in clinical studies, subjects may have difficulties to follow those guidelines. Moreover, suppressing eye movement or blinking may significantly distort brain activity $[18,19]$, since it requires attention from the subject.

\subsubsection{Artifact Rejection}

Alternatively, one often rejects corrupted epochs or trials, detected by visual inspection. Also this approach is problematic: first of all, this method results in loss of data, and therefore, it may not be applicable if the number of trials is low. Moreover, visual inspection is subjective and time consuming [20]. To overcome those issues, an automatic objective criterion is sometimes used; in particular, one often detects artifacts by means of a threshold: signals above a certain threshold are considered to be spurious. Unfortunately, such simple automatic criterion was shown not to be capable of detecting muscle artifacts [21].

Instead of rejecting epochs contaminated by artifacts, one may try to remove artifacts. Various approaches to artifact removal have been proposed:

- adaptive filtering [31, 32, 33], in particular Kalman filtering [34, 35],

- regression [36, 37, 38, 39],

- blind source separation (BSS; see, e.g., [40, 41, 42]).

In the following, we describe those three approaches to artifact removal.

\subsubsection{Adaptive Filtering}

Artifacts may be detected in frequency domain. However, the spectrum of artifacts is a priori unknown. Therefore, applying a fixed filter to EEG data would not be effective to remove artifacts; a fixed filter is highly effective to remove power supply signals, as we mentioned earlier, since those signals appear at a particular frequency $(50 \mathrm{~Hz}$ or $60 \mathrm{~Hz})$. To remove artifacts, on the other hand, the filter needs to adapt to the spectrum of the recorded EEG: it should attenuate the recorded EEG in frequency ranges that mostly contain artifacts $[31,32,33]$. For instance, instead of using an online notch filter centered at a fixed frequency, one may apply an offline notch filter whose characteristics are determined by the spectrum of the recorded EEG. One may additionally use EOG (electro-oculographic) or EMG measurements to design the adaptive filter, since those measurements are usually strongly correlated with artifacts. Adaptive filters can be designed in various ways; one approach is based on the assumption that EEG signals can be well described by a linear model [34, 35], in particular, an autoregressive-moving average model (ARMA); such model is 
fitted to the recorded EEG (using Kalman filtering and standard parameter estimation methods), and significant deviations from the model are considered as artifacts and are therefore eliminated.

Adaptive filters have two obvious drawbacks: they not only attenuate artifacts, but also affect the "wanted" signal, i.e., components of the recorded EEG that stem from brain activity; moreover, the EEG signals are assumed to be well-described by a linear system, which may not always be the case.

\subsubsection{Regression}

As we pointed out earlier, artifacts are often correlated with EOG or EMG channels. Regression may be used to quantify this correlation [36, 37, 38, 39], and to infer artifacts from EOG or EMG channels. The estimated artifacts are subtracted from the EEG signals. This approach has the following problem: EEG activity may propagate to EOG or EMG sensors (known as "bidirectional contamination"), and part of the EEG signal may therefore be removed by the subtraction procedure.

\subsubsection{Blind Source Separation}

An alternative approach, known as "blind source separation" (BSS; see, e.g., [40]), starts from the assumption that EEG signals can be described, to a good approximation, by a finite set of sources, located within the brain; each of those sources generate certain components of the EEG. Besides EEG, one sometimes also incorporates EOG and EMG signals into the analysis.

In the context of artifact rejection, one makes the additional assumption that artifacts are generated by a subset of the extracted sources; one removes those sources, and next reconstructs the EEG from the remaining "clean" sources. Not surprisingly, the key challenge is to determine what sources need to be removed.

BSS appears to be more suitable for artifact rejection than adaptive filtering and regression (see, e.g., [19, 20, 22, 41, 42, 43, 44]). The two main assumptions behind the BSS approach, i.e., both EEG and artifacts may be described by a limited number of sources, seem to good approximation to be fulfilled in the case of EEG recordings [21, 24]. As major advantage of BSS, artifacts within the EEG spectrum of interest can be removed without affecting the EEG, at least in principle [41].

Of course, as any method, BSS has its limitations. First of all, BSS is not fully automatic: one needs to visually inspect the components extracted by BSS and decide which components to remove; this time consuming process is not suitable for routine clinical EEG [41]. Furthermore, visual inspection is subjective [22], and the reliability of BSS is therefore limited. To limit the need for visual inspection, semi-automatic methods have been developed; those methods compute statistical markers to determine which BSS components should be removed [21, 43, 44]. Examples of such statistical markers are kurtosis, entropy, Hurst exponent, Renyi's entropy, time-frequency deviation, source scalp or EMG 
sensor location, and source amplitude. Semi-automatic artifact removal methods use more objective criteria and are hence more consistent; moreover, since they rely less on visual inspection, they tend to be much faster than techniques that require visual inspection. An exhaustive comparison of statistical markers has not been conducted yet, and consequently, it is unclear at this point which markers are the most appropriate for artifact detection.

\subsection{Preprocessing: Results and Discussion}

EEG recordings often contain unwanted signals which may bias the analysis of the EEG, and may lead to wrong conclusions; it is therefore necessary to eliminate or limit unwanted signals (artifacts) before analyzing EEG. We have reviewed various approaches to reduce artifacts: (i) one may try to suppress/avoid artifacts by instructing the subjects; (ii) alternatively, one may discard the trials with artifacts; (iii) one may try to remove artifacts by advanced signal processing methods such as adaptive filtering and blind source separation (BSS).

Artifact rejection methods seem generally to improve the separation between control subjects and $\mathrm{AD}$ patients, at different stages of the disease; in particular, a variety of studies report encouraging results for BSS methods [45, 46, 47, 48, $49,50]$.

We conclude this section with an important observation: EEG experts do not always agree on what the artifacts in a given EEG signal are; for example, in a recent EEG study [51], EEG experts only agreed in $75 \%$ of the cases. Therefore, removing all artifacts from EEG signals is virtually impossible. A probably more realistic objective, especially in studies of brain disorders, is to try to make sure that the EEG of different populations (e.g., control subjects and different types of patients) contain about the same amount of artifacts [43, 44]. Indeed, the results of the EEG analysis may be biased if one population contains substantially more artifacts than the other(s).

\section{Slowing of EEG}

As we mentioned earlier, one is often interested in specific frequency bands in the EEG, such as $4-8 \mathrm{~Hz}$ (theta), $8-10 \mathrm{~Hz}$ (alpha 1), $10-12 \mathrm{~Hz}$ (alpha 2), $12-30 \mathrm{~Hz}$ (beta), and $30-100 \mathrm{~Hz}$ (gamma) [30]. AD seems to affect those different frequency bands in specific ways. One of the major effects is EEG "slowing"; many studies have shown that Mild Cognitive Impairment (MCI) and Alzheimer's disease (AD) causes EEG signals to slow down (see, e.g., $[52,53,54,55,56,57,58,59,60,61,62,63,64,65]): \mathrm{MCI} / \mathrm{AD}$ is associated with an increase of power in low frequencies (delta and theta band, $0.5-8 \mathrm{~Hz}$ ) and a decrease of power in higher frequencies (alpha and beta, $8-30 \mathrm{~Hz}$ ). However, increased gamma band power $(30-100 \mathrm{~Hz})$ has been reported in MCI/AD patients compared to healthy age-matched control subjects [67].

To quantify the changes in spectral power, one usually applies Fourier transforms $[52,53,54,55,56,57,58,59,60,61]$ : one first computes the discrete 
Fourier transform $X(f)$ (see Fig. 1) at multiples of $f_{T}=1 / T$, where $T$ is the length of the signal. For example, if $T=20 \mathrm{~s}$ and the sampling frequency is $200 \mathrm{~Hz}$, one may compute the discrete Fourier transform at $0 \mathrm{~Hz}, 0.05 \mathrm{~Hz}, 0.1 \mathrm{~Hz}$, ..., 200Hz. According to Nyquist theorem, the discrete Fourier transform is affected by aliasing at frequencies larger than half the sampling frequency, and those values need to be discarded. In the previous example, one would only retain the discrete Fourier transform at $0 \mathrm{~Hz}, 0.05 \mathrm{~Hz}, 0.1 \mathrm{~Hz}, \ldots, 100 \mathrm{~Hz}$. In EEG analysis, however, one is often more conservative, and retains fewer frequency values, e.g., all frequencies up to one fifth of the sampling frequency [66]. In the same example, that would be $0 \mathrm{~Hz}, 0.05 \mathrm{~Hz}, 0.1 \mathrm{~Hz}, \ldots, 40 \mathrm{~Hz}$.

Next one computes the total power within each frequency band of interest, by summing the magnitude $|X(f)|$ of $X(f)$ over all integer frequencies within the frequency band; at last, one normalizes the power within each band by the total power, resulting in the relative power of each frequency band of interest.

Alternatively, one sometimes computes relative power by means of timefrequency maps $X(t, f)[62]$ (see Fig. 1); such map indicates how much energy of the signal is contributed by frequency $f$ at time instance $t$. Such maps are particularly useful if one is interested in the spectrum at a specific instance of time, for example, at a specific stage of a working memory or oddball task (see Section 7.4.1); such task was considered in [62], and therefore, time-frequency maps $X(t, f)$ were used in that study.

Interestingly, time-frequency maps of EEG signals are often sparse, as can be seen from Fig. 1: most energy is contained in specific regions of the timefrequency map ("bumps"), corresponding to transient oscillations. Recently, a procedure was proposed to extract such transient oscillations from timefrequency maps $[68,69,70]$; it was shown that transient oscillations in the EEG of MCI and AD patients occur more often at low frequencies compared to healthy control subjects $[63,64,65]$. In other words, those transient oscillations also exhibit slowing.

\section{Reduced Complexity of EEG Signals}

Several studies have investigated the complexity of EEG signals in MCI and AD patients. We will first review the complexity measures that have been used in those studies (Section 4.1); next we summarize the results from those studies (Section 4.2).

\subsection{Review of Complexity Measures}

Various complexity measures have been used to quantify EEG complexity; some of them stem from information theory [71, 72], including Tsallis entropy [72], approximate entropy [73], sample entropy [74], multiscale entropy [75, 76], automutual information (see, e.g., [77]), and Lempel-Ziv complexity [78, 79, 80, 81].

Entropy is a central quantity in physics and information theory. In information theory, entropy is a measure of the uncertainty associated with a random 


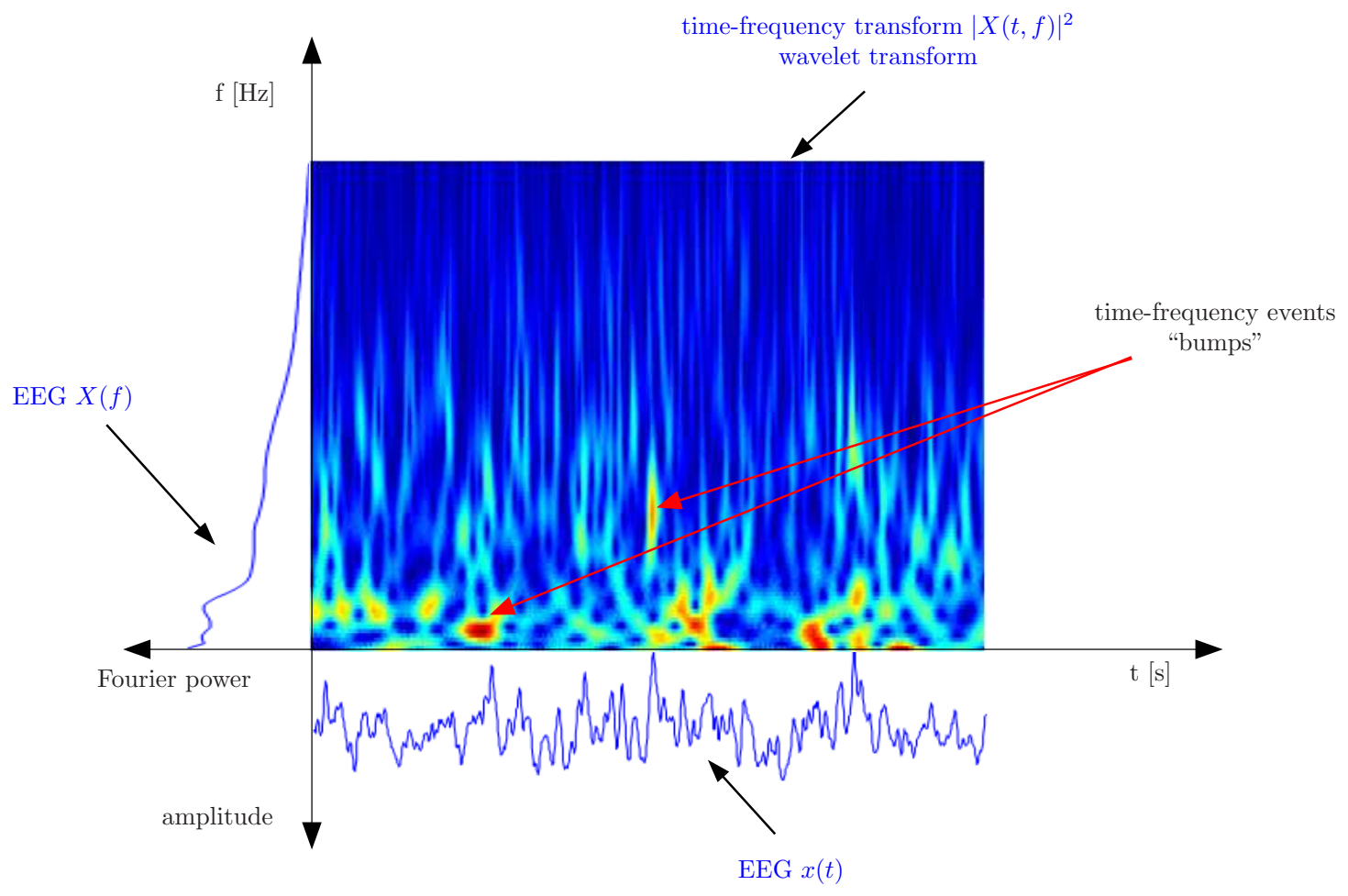

Fig. 1: Typical EEG signal, shown in time-domain $x(t)$, frequency domain $X(f)$, and time-frequency domain $|X(t, f)|$. As can be seen from this figure, time-frequency maps of EEG signals are often sparse: most energy is contained in specific regions of the time-frequency map ("bumps"), corresponding to transient oscillations. 
variable; this formulation of entropy was introduced by Shannon [71] (see [72] for an excellent textbook on information theory), and is often referred to as "Shannon entropy". Tsallis entropy is a family of entropy measures parameterized by a real number $q[72]$. Tsallis entropy reduces to Shannon entropy for $q=1$. The parameter $q$ is a measure of the non-extensitivity of the system of interest: the Tsallis entropy of two independent systems is in general not equal to the sum of the Tsallis entropies of those systems; only when $q=1$ the Tsallis entropy of two independent systems is equal to the sum of the Tsallis entropies of those systems, as expected for an extensive system. In [82] the Tsallis entropy of EEG signals is determined by quantizing the amplitude of the EEG; Tsallis entropy is computed from the histogram of amplitude values (with $q=0.5$, for the sake of definiteness).

Approximate entropy [73] reflects the likelihood that patterns in a given signal will not be followed by additional "similar" patterns. Sample entropy [74] is a refinement of approximate entropy that it designed to have smaller bias.

The idea behind multi-scale entropy $[75,76]$ is simple: consecutive coarsegrained time series are constructed from the original time series, with increasing coarseness parameter $\tau$; the entropy of each resulting time series is computed. To this end, one may apply approximate, sample, or Tsallis entropy. As a result, one has determined how entropy of the time series depends on the time scale $\tau$. By averaging the entropy over several values of $\tau$, a multi-scale estimate of the entropy may eventually be obtained.

Auto-mutual information of a time series (see, e.g., [77]) is the mutual information between a time series and its time-shifted copies; note that the time series is supposed to be stationary. The faster auto-mutual information decays with time lag, the more complex the time series is considered to be.

Lempel-Ziv complexity [78] counts the number of different patterns in a signal; the less such patterns, the better a signal may be compressed. The well-known Lempel-Ziv-Welch compression algorithm is derived from that basic idea $[79,80,81]$. The extent of compression can be computed as the ratio of the file size before and after compression. The compression rate is a measure of the regularity of the signal.

Some complexity measures have their roots in physics: fractal dimension [83], correlation dimension [84], and largest Lyapunov exponent [85, 86]. Fractal dimension is a statistical quantity that indicates how completely a fractal appears to fill space, as one zooms down to finer and finer scales. A fractal is generally a fragmented geometric shape that can be split into parts, each of which is (at least approximately) a reduced-size copy of the whole [83]. Natural objects that approximate fractals to a degree include clouds, mountain ranges, lightning bolts, coastlines, and snow flakes. There are several definitions of fractal dimension; correlation dimension [84] is one of them, other definitions are closely related to entropy, and are information-theoretic in nature.

The Lyapunov exponent of a dynamical system is a quantity that characterizes the rate of separation of infinitesimally close trajectories $[85,86]$; it is noteworthy that Lyapunov exponents are closely related to the entropy of the dynamical system [86]. The maximal Lyapunov exponent determines the 
predictability of a dynamical system. A positive maximal Lyapunov exponent usually indicates that the system is chaotic.

\subsection{Overview Of Results}

As pointed out earlier, several studies have investigated whether the complexity of EEG signals is perturbed by MCI or AD [77, 82, 87, 88, 89, 90, 91, 92, $93,94,95]$. The following measures have been applied in this context: approximate entropy [87, 88, 89, 90], sample entropy [87, 94], Tsallis entropy [82], multiscale entropy [87, 95], auto-mutual information [77, 87, 88], Lempel-Ziv complexity [87], universal compression [82], fractal dimension [91], correlation dimension [92, 93], and largest Lyapunov exponent [93].

Interestingly, those studies all observed that the EEG of MCI and AD patients seems to be more regular (and, equivalently, less complex) than of agematched control subjects. As far as we know, no study has identified the underlying biological phenomenon. We conjecture that due to the MCI/AD induced loss of neurons and perturbed anatomical and/or functional coupling, fewer neurons interact with each other, and the neural activity patterns and dynamics become simpler and more predictable. Moreover, it is possible that the MCI/AD induced phenomenon of reduced complexity is related to slowing, since "slower" (lowpass) signals are intrinsically more regular.

\section{Perturbations in EEG Synchrony}

Numerous studies have reported decreased EEG synchrony in MCI and AD patients under rest conditions ("spontaneous EEG"): the statistical dependence between spontaneous EEG signals recorded from different channels seems to be generally lower in MCI and AD patients than in age-matched control subjects.

In those studies, a large variety of synchrony measures have been applied, e.g., Pearson correlation coefficient [96, 97, 98], coherence [96, 97, 98, 99, 100, 101, 102, 103, 104, 105, 106, 107], Granger causality [96, 97, 98, 108, 109], information-theoretic [77, 96, 97, 98] and state space based synchrony measures $[55,96,97,98,110,111,112,113,114,115,116]$, phase synchrony indices $[55,96,97,98,107,117,118]$, and stochastic event synchrony [96, 97, 98, $119,120,121,122]$.

In the following, we will briefly review some of those synchrony measures (Section 5.1); next we describe results of studies that have applied those measures to the EEG of MCI and AD patients (Section 5.2).

\subsection{Review of Synchrony Measures}

Many different measures of synchrony have been employed in the physical sciences, signal processing and in the study of neurobiology (see, e.g., [123, 124, 125]). Many of these techniques have overlapping properties while some are substantially different. All seek to quantify the relationship between two signals or sensors and, by extension, the sources they represent. 
Probably the most basic synchrony measure is the Pearson correlation coefficient; it quantifies linear correlations between pairs of signals. The (magnitude) coherence function is an extension of the correlation coefficient from time-domain to frequency domain; it measures linear correlations in frequency domain [125]. Non-linear extensions of the correlation coefficient have been proposed (see, e.g., [126]). Granger causality extends the correlation coefficient from pairs of signals to multiple signals [127]. It allows us to estimate the causality of linear interactions. Examples of Granger measures are directed transfer function (DTF) and partial directed coherence (PDC); at least ten other linear Granger measures have been proposed in the literature [127]. In addition, nonlinear extensions of Granger causality have recently been developed (see, e.g., $[128,129])$.

The approaches mentioned so far use information about the amplitude ("magnitude") of the signals ("magnitude synchrony"). Alternatively, one may investigate correlations between the phase of signals [130]. Indeed, the instantaneous phase of different signals may be strongly synchronized even if the amplitudes of those signals are statistically independent. Examples of such measures are mean phase coherence [130], phase lag index [107], imaginary coherence [131], and global field synchrony (GFS) [132]; the first three measures are only applicable to pairs of signals, the latter allows us to detect phase coupling between a large number of signals.

An interesting alternative family of synchrony measures, referred to as state space based synchrony or generalized synchrony, stems from physics $[123,133]$. The signals at hand are assumed to be generated by some (unknown) deterministic, potentially high-dimensional, non-linear dynamical system. As a first step, one tries to reconstruct that system, by representing the signals in a state space: each signal is represented as a trajectory in that space. Signals are considered to be synchronous if their trajectories remain close to each other. Examples of state space based synchrony measures are synchronization likehood [133], omega complexity [134], the S-estimator [135], and the S-H-N-indices [124].

Yet another approach draws inspiration from information theory [72]: one treats the given signals as stochastic processes and calculates classical similarity and distance measures for such processes, e.g., mutual information and Kullback-Leibler divergence, in time domain (see, e.g., [136]) as well timefrequency domain $[137,138]$ (see also [139, 140]).

An entirely different approach has been developed recently, referred to as stochastic event synchrony (SES) [119, 120, 121]; it characterizes the interaction between certain events in signals. In brain signals, those events can be spikes or transient oscillatory components (see Section 3 and Fig. 1). Those two types of events are considered in [119, 120, 121].

Many other synchrony measures have been developed; the above list contains most common and some recent approaches to determine synchrony, but it is by no means exhaustive.

In a recent study, it was shown that many classical and recently proposed similarity measures, although developed from different perspectives, lead to similar numerical values [96, 97] (at least for one particular EEG data set). A total 
of 35 similarity measures were applied to normal EEG, and the correlation between those measures was determined. Many measures are strongly correlated (either positively or negatively). As a consequence, distinct families of synchrony measures may be identified. This result suggests that it is not necessary to apply 35 or more synchrony measures; it probably suffices to combine measures from each family.

\subsection{Overview Of Results}

\subsubsection{Pearson Correlation Coefficient}

The Pearson correlation coefficient, albeit a simple and well-understood measure, does not seem to be often used to determine EEG synchrony in MCI and $\mathrm{AD}$; a recent study [97] reports decrease in Pearson correlation in the EEG of MCI patients compared to healthy age-matched subjects, however, it was not statistically significant. No effect was found in AD patients compared to healthy age-matched subjects [98].

\subsubsection{Magnitude and Phase Coherence}

A large number of studies have reported decreased magnitude and phase coherence in the EEG of MCI and AD patients [99, 100, 101, 102, 103, 104, 105, 106, $107,141,142]$. In [100] those measures even allowed to separate depressed MCI patients from healthy age-matched subjects (control subjects). In some other studies, however, no significant effects on coherence was observed, neither between AD and controls [98, 111, 133] nor between MCI and controls [96, 97, 143].

\subsubsection{Granger Causality}

Various (linear) Granger measures have been applied to the EEG of MCI [97] and AD patients [98]. The full frequency directed transfer function (ffDTF) was significantly reduced in MCI [97] and AD patients [98] in comparison to control subjects, whereas DTF was only significantly reduced in AD patients [98]. In [109] directed transfer function (DTF) was applied to the EEG of amnesic MCI and AD patients; it was found that the parietal to frontal direction of the information flux was weaker in MCI and AD subjects, specifically for alpha and beta rhythms.

\subsubsection{Phase Synchrony}

Decreased phase synchrony has been reported in EEG of patients with mild AD (resting condition), using three different measures: phase coherence, phase lag index (PLI), and imaginary component of coherence [107]. Koenig et al. [117] observed a general decrease of global field synchrony (GFS) in correlation with cognitive decline and AD. In [97], however, GFS did not manifest this effect in MCI. Besides GFS, five alternative phase synchrony measures were investigated in that study; only one of them indicated a decrease in phase synchrony, but 
it was not significant after post-correction. In a similar study [98], no effect was found in AD patients. Recently, the spatial distribution of EEG phase synchrony in AD patients has been investigated [144]; robust differences in that distribution were found between $\mathrm{AD}$ patients and healthy subjects.

\subsubsection{State Space Synchrony}

Using state space based synchrony methods, significant loss of EEG synchrony was found in MCI [111] and AD patients [14, 111, 112, 113, 114, 115, 116, 145], which evolved consistently from MCI to AD stage [111]. In [97] the indices N and S, and the S-estimator were lower in MCI patients, however, the effect was not significant after Bonferroni correction. The index $\mathrm{S}$ was found to be significantly lower in AD patients [98]. In a related study [110], the index H was significantly lower in MCI and AD patients, however, no such effect was observed for S. Omega complexity was found to be increased in patients with mild AD, which corresponds to decreased synchronization [146]; that effect was also observed in MCI patients [97], but it was non-significant.

\subsubsection{Information Theoretic Measures}

The mutual information between different EEG channels was observed to be significantly lower in AD patients than in control subjects [77]. No significant effects were found in MCI patients [97], using mutual information (in time domain as well as time-frequency domain), Kullback-Leibler divergence, and other divergence measures (in time-frequency domain).

\subsubsection{Stochastic Event Synchrony}

The stochastic event measures have been applied to the EEG of MCI [97] and AD patients [97]. In both studies, a significant reduction in EEG synchrony was found compared to healthy age-matched subjects; stochastic event synchrony (SES) and a Granger measure (ffDTF; see Section 5.2.3) were used as features in discriminant analysis: MCI [97] and AD patients [97] could be fairly well separated from healthy control subjects.

\subsubsection{Graph-Theoretic Methods}

Synchrony measures may be used to construct macroscopic network models of the brain, particularly, from EEG signals; for example, each node in the network may correspond to an EEG channel (electrode), and one may draw an edge between a pair of nodes if the synchrony value between the EEG of the corresponding channels is above a certain threshold. The properties of the resulting networks may be analyzed through graph theory, in particular, the theory of small-world networks [147]. By means of that theory, several studies have shown that EEG signals of MCI and AD patients have weaker small-world network characteristics compared to age-matched control subjects [148, 149]. 


\subsubsection{Increased Synchrony during Working Memory Task}

The results we have discussed so far in Section 5.2 are mostly for EEG recorded under rest conditions. Some researchers have also recorded EEG of MCI and AD patients while they perform certain tasks (see Section 7.4.1). More specifically, in some studies that investigate the EEG of MCI and AD patients performing working memory tasks $[143,150,151]$, an increase of EEG synchrony was observed. This inverse effect is often interpreted as the result of a compensatory mechanism in the brain; magnitude coherence was applied as a measure for EEG synchrony. In [114] a similar effect was observed in MCI patients by means of the synchronization likelihood measure: one observed an increase of EEG synchrony during working memory task, but there was no significant effect in rest condition.

\subsection{Perturbations in EEG Synchrony: Discussion}

Numerous studies have reported decreased EEG synchrony in MCI/AD patients. However, as our review has shown, there is no consensus yet as to what synchrony measures are the most effective in the context of diagnosing AD. The main problem is that most studies use just one measure or very few measures, and many of those studies analyze different data sets; consequently, it is difficult to rigorously compare the various measures. Moreover, as we pointed out earlier, it has been shown recently that many classical and recently proposed similarity measures lead to similar numerical values [96, 97] (at least for one particular EEG data set), and that several families of synchrony measures may be identified. Therefore, we suggest to apply synchrony measures from each of those families (e.g., Pearson correlation coefficient, Granger measures, phase synchrony, state space synchrony, and stochastic event synchrony) to EEG data of AD patients. Such studies may eventually provide us a clearer picture of what measures are most relevant for diagnosing AD.

The observed loss in synchrony in $\mathrm{AD}$ is often attributed to a functional disconnection of the neocortex; it is probably not simply due to a loss of cortical neurons. For example, it may result from anatomical disconnections among different cortical regions in combination with reduced cholinergic coupling between cortical neurons [77]. In particular, a common hypothesis is that basal forebrain neurons may be severely affected in AD, resulting in a cerebral cholinergic deficit that leads to memory loss and other cognitive symptoms [77]. In other words, not solely loss of neurons but also perturbed anatomical and/or functional coupling may result in impairments in the temporal coordination of distributed neuronal activity [152], which in turn may lead to the cognitive disturbances associated with AD.

One should keep in mind, however, that it is hard to directly interpret results obtained with synchrony measures. Synchrony measures obtained from EEG signals may be significantly affected by brain events other than changes of synchrony, and by choices (like the reference electrodes) that necessary have to be made during the analysis. Furthermore, as a single active source in the 
brain may affect the EEG signals across the entire scalp, changes in synchrony, and especially simultaneity of some events across channels, may be observed when the activity of one source alone changes, which is remote from a change in synchrony.

Interestingly, various studies have constructed source models from the EEG of MCI and AD patients [108, 153, 154, 155, 156, 157, 158, 159, 160]. We will discuss some of those studies later on in this paper.

\section{Discussion: Where Are We standing?}

As pointed out earlier, EEG seems an attractive brain imaging modality for diagnosing AD, since EEG recording systems are relatively inexpensive and (potentially) mobile. Moreover, in contrast to most other non-invasive brain imaging methods, EEG has high temporal resolution, and may as a consequence contain crucial information about abnormal brain dynamics in $\mathrm{AD}$ patients. In particular, three major effects of AD on EEG have been observed: slowing of the EEG, reduced complexity of the EEG signals, and perturbations in EEG synchrony; an overview of the studies reviewed in Sections 3 to 5 is given in Table 1.

Numerous studies have investigated the potential of EEG as a diagnostic tool for AD. At present, however, it is hard to assess whether EEG is truly useful for diagnosing AD. First of all, most studies report the results of statistical tests (p-values) without statistical post-correction. Since typically one conducts multiple tests simultaneously (sometimes hundreds or even thousands), e.g., individual pairs of electrodes or frequency bands, it is important to eliminate false positives. To this end, one may apply Bonferroni post-correction [161], or more powerful alternatives, such the false-discovery-rate correction method of Storey [162]. Unfortunately, not all studies on diagnosing AD from EEG signals apply such post-correction methods, and therefore, it is not always obvious how to interpret the reported results.

Second, few studies conduct discriminant analysis (linear or non-linear discriminant analysis, using support vector machines, neural networks, etc.; see [163] for a study that tests various classification algorithms for diagnosing AD). A major challenge is to obtain a reliable estimate of the classification error. Many studies that do conduct discriminant analysis report unreliable estimates of the classification error, since the classifiers are trained and tested with the same data set. The classifiers may be overfitted to the data at hand; as a consequence, the reported classification results may not generalize to other data sets, and hence, they may be overoptimistic. To obtain more reliable classification results, one may for example apply crossvalidation, as has indeed been done in a handful studies (e.g., [45, 53, 62, 94, 97, 163, 164, 165]). However, crossvalidation only yields reliable classification rates if the data set is sufficiently large and the classifiers have a limited number of parameters. To obtain more reliable classification results, one should ideally use three independent data sets:

- The first data set is used to train various classifiers. 


\begin{tabular}{|c|c|c|}
\hline Phenomenon & Approach & References \\
\hline Slowing & $\begin{array}{c}\text { Fourier power } \\
\text { Time-frequency map } \\
\text { Bump models }\end{array}$ & $\begin{array}{c}{[52,53,54,55,56,57,58,59,60,61]} \\
{[62]} \\
{[63,64,65]}\end{array}$ \\
\hline Reduced complexity & $\begin{array}{c}\text { Approximate entropy } \\
\text { Sample entropy } \\
\text { Tsallis entropy } \\
\text { Multiscale entropy } \\
\text { Auto-mutual information } \\
\text { Lempel-Ziv complexity } \\
\text { Universal compression } \\
\text { Fractal dimension } \\
\text { Correlation dimension } \\
\text { Largest Lyapunov exponent }\end{array}$ & $\begin{array}{c}{[87,88,89,90]} \\
{[87,94]} \\
{[82]} \\
{[87,95]} \\
{[77,87,88]} \\
{[87]} \\
{[82]} \\
{[91]} \\
{[92,93]} \\
{[93]}\end{array}$ \\
\hline Perturbed synchrony & $\begin{array}{l}\text { Pearson correlation coefficient } \\
\text { Magnitude/phase coherence } \\
\text { Granger causality } \\
\text { Phase synchrony } \\
\text { State space synchrony } \\
\text { Information theoretic measures } \\
\text { Stochastic event synchrony } \\
\text { Graph-theoretic methods }\end{array}$ & $\begin{array}{c}{[96,97,98]} \\
{[96,97,98,99,100,101,102,103,104,105,106,107]} \\
{[96,97,98,108,109]} \\
{[55,96,97,98,107,117,118]} \\
{[55,96,97,98,110,111,112,113,114,115,116]} \\
{[77,96,97,98]} \\
{[96,97,98,119,120,121,122]} \\
{[147,148,149]}\end{array}$ \\
\hline
\end{tabular}

Tab. 1: Overview of studies concerning the three major effects of MCI/AD on EEG: slowing of the EEG, reduced complexity of the EEG signals, and perturbations in EEG synchrony. The studies are described in detail in Sections 3 to 5 . 
- The resulting classifiers are evaluated on the second data set; one retains the classifier with the best classification results on the second data set.

- The latter classifier is then evaluated on the third data set.

The classification results on the third data set are a reliable estimate of the actual classification performance, as long as the three data sets are sufficiently large and independent.

Third, in most existing studies, a single measure to detect EEG abnormalities is applied to a single EEG data set. Since almost every study considers a different measure and a different EEG data set (but see, e.g., $[156,163]$ ), it is hard to compare existing studies and to verify whether results are consistent.

Fourth, it is likely that one will need to combine various EEG characteristics in order to obtain a good diagnostic tool for AD, e.g., based on slowing, loss in EEG synchrony and complexity, and other features yet to be discovered. However, few studies systematically investigate large collections of EEG features (e.g., [53, 97]); it would be of great interest to apply dimensionality reduction methods to hundreds or even thousands of EEG features, to determine the most discriminative EEG features in a disciplined and statistically rigorous fashion. Moreover, it still needs to be verified whether the effects listed in Sections 3 to 5 are independent. For example, it may be that EEG slowing and loss of EEG complexity are two sides of the same coin.

At last, most EEG studies (if not all) on the diagnosis of MCI/AD are retrospective: they are based on the medical diagnosis of $\mathrm{AD}$, and the subsequent analysis of two (or more) patient populations, i.e., one population medically diagnosed with AD or MCI and the other medically diagnosed as healthy. The EEG studies then classify the patients based on EEG metrics, and claim to have built a diagnostic tool. As far as we know, no studies so far use EEG in a predictive way to actually diagnose patients, and verify the results with medical diagnosis later.

\section{Remaining Challenges and Topics for Future Research}

In this section, we point out several remaining challenges and topics for future research.

\subsection{Bottleneck: EEG Databases of AD patients}

At present, it is fairly difficult to gain access to EEG data of MCI or AD patients. Such databases are not publicly available, in contrast to ECG (electrocardiogram) and other biomedical data (e.g., [166]). As a result, it is hard to systematically benchmark and assess the existing methods for diagnosing AD from EEG signals. A freely accessible repository for anonymized EEG data (perhaps organized by NIH or similar organizations) may be a solution to this problem. 


\subsection{Relation Between EEG Markers and Cognitive Decline}

The precise relation between EEG abnormalities and the decline of cognition and memory in AD patients remains largely unexplored. A few recent studies address this issue; we outline some of the results.

\subsubsection{Overview of Recent Results}

It was observed in [61] that the theta/gamma ratio of relative EEG power is significantly associated to memory decline in MCI patients. Similar results have been obtained in [54] for MCI and AD patients: theta relative power and alpha reactivity (i.e. loss in alpha power during cognitive task as compared to eyes closed) seem to be related to decreased performance in various cognitive domains (global cognition, language, memory and executive functioning); alpha coherence, however, does not seem to correlate with cognitive decline. In a related study [118], it was observed that GFS values of the EEG of AD patients are positively correlated with MMSE and CDR scores, which are both measures of cognitive performance.

In [155], cortical sources were extracted from resting eyes-closed EEG of MCI and $\mathrm{AD}$ patients, by means of low-resolution brain electromagnetic tomography (LORETA); the cortical sources of resting delta and alpha rhythms seemed to correlate with neuropsychological measures of immediate memory, in particular, corsi span forward (visuo-spatial immediate memory probing focused attention) and digit span forward (immediate memory for digits probing focused attention).

Another study [167] deals with the rate of cognitive decline, i.e., the rate at which cognition declines over the years. More precisely, it investigates the relation between rate of cognitive decline in SMC (subjective memory complaints), MCI and AD patients, and its relation to EEG markers; it was found that the rate of cognitive decline increased between groups: AD showed advanced decline, and SMC/MCI groups represented intermediate stages of decline relative to normal aging expectations. Substantial perturbations in the EEG of AD patients were observed over age, coupled with memory decline: EEG slowing and decreased working memory P450 component. The MCI group, however, showed less severe cognitive changes but loss in the working memory N300 component and slow-wave (delta) EEG, associated with decline in memory. Interestingly, in this study the EEG markers in MCI and AD patients seem to be quite distinct, although the cognitive decline rate progresses continuously between both groups [167]; this is strong contrast with [155], where the EEG markers changed continuously between MCI and AD patients.

\subsubsection{Cognitive Decline and EEG: Discussion}

A handful of studies have shown that certain EEG markers seem to correlate with decreased performance in various cognitive domains. More research is required though before any conclusions can be drawn.

Those observations also seem to suggest that EEG markers may help to separate MCI from AD. Such differential diagnosis is important, since potential 
therapies and treatments may differ depending on the disease (MCI vs. AD). Several recent studies have assessed the potential of EEG for distinguishing MCI from AD patients (see [116, 154, 164, 168, 169, 170, 171, 172, 173, 174]), some of which we will discuss later on. Along similar lines, a few studies have investigated whether EEG allows us to separate MCI patients that develop AD (Progressive MCI; PMCI) from MCI patients that do not develop AD (Stable MCI; SMCI) [168, 169, 189]; those studies are discussed in Section 7.4.4 and 7.4.5. Although such studies are very promising, more research in this direction is required in order to assess whether EEG may be used to differentiate MCI from AD patients, and SMCI from PMCI patients.

\subsection{Differential Diagnosis of Dementia}

It is also of great importance to verify whether EEG helps to distinguish between $\mathrm{AD}$ and other dementias; in the last years, studies have investigated whether EEG alone allows to differentiate between AD, subjective memory complaints [116, 173, 175], psychiatric disorders [173], vascular dementia [173, 176, 177, 178, 179, 180], fronto-temporal dementia [116, 173, 180], dementia with Lewy bodies [173, 181, 182], dementia with Parkinson's disease [182], and vascular dementia with Alzheimer's disease [60]. The preliminary results are generally promising, but surely, additional investigations will be required to explore the potential of EEG for differential diagnosis of dementia, and brain disorders at large.

\subsection{Design of EEG Experiments}

An important degree of freedom is the EEG recording condition. One may record EEG:

- while the subject is at rest awake (with open or closed eyes) or asleep,

- while the subject is being stimulated with auditory, visual, tactile, or other signals,

- while the subject performs a task, e.g., an oddball or working memory task (see Section 7.4.1).

Such stimulation protocols may be used to probe specific brain circuits supposedly disrupted AD, and therefore, they may make EEG even more sensitive to brain pathologies related to $\mathrm{AD}$.

Most EEG studies of MCI and AD conduct recordings in resting condition. Several recent studies consider alternative EEG recording conditions, e.g., during stimulation with light or during oddball or working memory tasks. In the following, we will review some results of those studies (Section 7.4.2 to 7.4.5), after reminding the reader of some basic terminology (Section 7.4.1); next we point out important open problems in this research area (Section 7.4.6). 


\subsubsection{Relevant Basic Terminology}

An evoked potential (or "evoked response"; EP) is an electrical potential recorded from the nervous system of a human or other animal following presentation of a stimulus, as distinct from spontaneous potentials [183].

An event-related potential (ERP) is any measured brain response that is directly the result of a thought or perception [184]. More formally, it is any stereotyped electrophysiological response to an internal or external stimulus. The major ERP components are given distinct names: they are referred to by a preceding letter indicating polarity $(\mathrm{N}$ or $\mathrm{P}$ ) followed by the typical latency in milliseconds. Thus, the N400 ERP component is described as a negative voltage deflection occurring approximately 400ms after stimulus onset, whereas the P600 component describes a positive voltage deflection $600 \mathrm{~ms}$ after stimulus onset. The stated latencies for ERP components are often quite variable; for example, the N400 component may exhibit a latency between 300ms and 500ms.

It is noteworthy that evoked potentials and event-related potentials are quite different: While evoked potentials reflect the processing of the physical stimulus, event-related potentials are caused by the "higher" processes, that might involve memory, expectation, attention, or changes in the mental state, among others [184].

In an oddball task, subjects respond to target stimuli that occur infrequently and irregularly within a series of standard stimuli. Detection of these targets reliably evokes transient activity (ERP).

In a working memory task, subjects are tested whether they can memorize ("encode") and recall ("retrieve") certain stimuli, e.g., images or letters. A classical example is the $n$-back task: The subject is presented with a sequence of stimuli, and the task consists of indicating when the current stimulus matches the one from $n$ steps earlier in the sequence. The load factor $n$ can be adjusted to make the task more or less difficult. For example, an auditory three-back test $(n=3)$ could consist of the experimenter reading the following list of letters to the test subject:

\section{T L H C H S C C Q L C K L H C Q T R R K C H R.}

The subject is supposed to indicate when the letters marked in bold are read, because those correspond to the letters that were read three steps earlier.

We will now discuss some recent studies that have considered sleep EEG (Section 7.4.2), visual and auditory EP/ERP (Section 7.4.3 and 7.4.4 respectively), and working memory ERP (Section 7.4.5) in MCI and AD.

\subsubsection{Sleep EP in $\mathrm{MCl}$ and $\mathrm{AD}$}

To our knowledge, only one study has investigated evoked potentials during sleep in $\mathrm{AD}$ patients [185]. In the latter, EEG data were recorded during stage 2 non-REM sleep, which is characterized by sleep spindles and K-complexes. Tone clicks were presented binaurally, which sometimes evoked K-complexes; it was observed that $\mathrm{AD}$ patients produced significantly fewer evoked K-complexes, 
and had substantially smaller N550 amplitudes than control subjects. Moreover, a lower probability of eliciting a K-complex correlated with greater dementia severity, as measured by the MMSE and Dementia Rating Scale. Interestingly, an earlier study [187] with young and elderly healthy subjects had shown that there were significant reductions in evoked K-complex probability and N550 amplitude and a significant increase in N550 latency, as a function of age. Both studies are therefore in agreement with theories that consider AD to represent an intensified form of the process of aging.

\subsubsection{Visual EP and ERP in $\mathrm{MCl}$ and $\mathrm{AD}$}

The potential of visual EPs for diagnosing AD was assessed in [186]. Stationary pattern onset EPs, visual motion onset EPs, and behavioral ERPs (during centered visual fixation) were recorded in AD patients and healthy control subjects. Psychophysical assessment demonstrated visual motion perceptual impairments in AD patients, half of whom also showed low sensitivity in the attentional task. The low sensitivity AD patients had small pattern onset and absent motion onset EPs, whereas the high sensitivity AD patients had large pattern onset EPs and normal motion onset EPs; visual EPs hence seemed to be abnormal in all $\mathrm{AD}$ patients.

In the experiments of [174], EEG of MCI and AD patients, and control subjects was recorded while subjects were passively viewing a visual stimulus. EEG source reconstruction revealed robust differences in the source locations in those three groups.

Event related EEG coherence in patients with $\mathrm{AD}$ and control subjects was analyzed in [142], using a visual oddball paradigm. The AD patients showed lower values of evoked coherence.

\subsubsection{Auditory EP and ERP in $\mathrm{MCl}$ and $\mathrm{AD}$}

Two recent studies $[62,188]$ consider auditory ERP (AERP) in AD patients, using an auditory oddball paradigm. In [188] the N200 and P300 latency were found to be significantly prolonged in AD patients, but there were no differences in amplitude. Moreover, differences were observed in stimulus-related responses in the delta band: a significant enhancement of the delta response occurred in healthy subjects (especially at the frontal location), whilst this delta reactivity was not detectable in AD patients. In [62] various types of wavelets were used to extract features from ERP recorded during auditory oddball tasks; those features were then exploited in discriminative analysis with leave-one-out crossvalidation. Consequently, (probable) AD patient patients could be separated fairly well from healthy control subjects. Interestingly, especially the AERPs induced by novel tones (as opposed to target tones) were discriminative.

Along the same lines, AERPs of MCI and AD patients, and healthy subjects were studied in [170]. As in [188], latencies of N200 and P300 components were 
prolonged in patients compared to controls; using those latencies, one was able to separate the three different subject groups fairly well.

Using a similar auditory oddball paradigm as in [62, 170, 188], it was investigated in [189] whether latencies and amplitudes of the major waves of AERP correlate with memory status of patients with MCI and with conversion to AD. Latencies of all major AERP components were prolonged in patients compared to controls. The N200 amplitudes were significantly higher in patients, but no significant differences were observed in P300 amplitudes. Based on N200 latency, one could distinguish MCI patients that converted to AD from MCI patients that had not converted. However, MMSE scores of patients did not correlate with latency or amplitude of the AERP components.

Similar results were reported in $[156,190,191]$. In [190] P300 components in MCI and AD patients and healthy control subjects were analyzed, with an auditory oddball paradigm. Subcomponents of the P300, i.e., P3a and P3b, were determined by dipole source analysis, using the Brain Electric Source Analysis software (BESA) [192]. AD patients were characterized by a diminished P3b amplitude and longer P3a latency and reaction time. Those results were later replicated on a different EEG data set [156]. In [191] the P300 component of ERP was investigated in AD patients and control subjects, but now using a visual and auditory oddball paradigm. All subjects performed four oddball tasks that varied systematically in task difficulty. The P300 amplitude was smaller and the peak latency was longer for the AD patients across tasks and modalities. P300 differences between AD patients and controls were largest for the relatively easy tasks, with little influence of stimulus modality observed.

The relation between P300 latency and response speed in MCI and AD was investigated in [193], again using an auditory oddball paradigm. The P300 amplitude and latency distinguished the groups and showed a significant correlation with response speed.

The aim of [194] was to investigate whether steady state EPs are relevant for diagnosing AD. More precisely, $40 \mathrm{~Hz}$ auditory steady state EPs were recorded in MCI and AD patients, and healthy control subjects. The results showed a significant increase of $40 \mathrm{~Hz}$ SSR power in the AD group compared to MCI and controls. Furthermore a moderate correlation between $40 \mathrm{~Hz}$ steady state EP power and cognitive performance was shown.

\subsubsection{Working Memory Tasks in $\mathrm{MCl}$ and $\mathrm{AD}$}

In [195] event-related desynchronization (ERD) and synchronization (ERS) was studied in MCI and AD patients during a working-memory task; the $1-20 \mathrm{~Hz}$ frequency band was analyzed. The AD patients were reported to make more errors than the controls and the MCI group. During encoding of the memory set, ERD in the $10-20 \mathrm{~Hz}$ band occurred in the MCI group, but not (or less) in the controls or AD patients. During retrieval, ERD in the $7-17 \mathrm{~Hz}$ occurred in MCI patients and controls, but was absent in the AD patients.

As in [189], the aim of [168] was to find ERP signatures that correlate with 
conversion from MCI to AD. Patients with amnestic MCI were longitudinally studied annually with an ERP paradigm in which semantically congruous and incongruous target words are repeated after initial presentation. When congruous category-exemplars are repeated, smaller P600s (relative to initial presentation) are normally elicited; repetitions of semantically incongruous words yield normally smaller N400 amplitude. Interestingly, those effects were observed in the non-converting MCI patients but not (or less) in the MCI patients that converted to AD. Abnormalities of the P600 or N400 in MCI seem therefore to be associated with an increased risk of subsequent conversion to AD. The same ERP paradigm was also used in [196]; it was shown there that normal N400 and $\mathrm{P} 600$ word repetition effects were absent in $\mathrm{AD}$ patients.

The potential of ERP to predict conversion from MCI to AD was also explored in [169]. To this end, MCI and AD patients, and healthy control subjects performed a working memory task. At 1 year follow-up, about half of the MCI patients showed progressive cognitive decline (PMCI), the others remained stable (SMCI). The P200 and N200 ERP latency and beta event-related synchronization (ERS) were analyzed. Both P200 and N200 latencies were longer in PMCI and AD patients compared with healthy subjects and SMCI patients, and beta ERS was of lower amplitude. Similar results were obtained in [172], using the same ERP paradigm; besides the working memory task, the subjects also performed a control task. The ERP of the control task was subtracted from the ERP of the working memory task; the resulting component $\mathrm{P}_{\mathrm{wm}}$ was significantly larger than baseline in SMCI patients, but much weaker (or entirely absent) in PMCI patients; component $\mathrm{P}_{\mathrm{wm}}$ therefore seems to discriminate between SMCI and PMCI.

In [165], working memory ERPs of AD patients and healthy control subjects were investigated, using principal component analysis (PCA); the latter allows to decompose the ERPs as superpositions of elementary scalp voltage components. The ERP components extracted through PCA allowed, to some extent, to separate $\mathrm{AD}$ patients from healthy control subjects.

\subsubsection{Design of EEG Experiments: Discussion}

In summary, various studies have demonstrated changes in ERP and ERS in MCI/AD patients. The main three effects are: (i) larger ERP latencies; (ii) smaller ERP amplitudes; (iii) smaller ERS. As far as we know, no study has identified the underlying biological factors for those three observations. However, one may conjecture that the brain becomes slower and weaker to respond since neurons and synapses are damaged due to AD: it becomes more difficult and it takes more time for information to propagate from one brain area to an other. In other words, loss of neurons but also perturbed anatomical and/or functional coupling may result in impairments in the temporal coordination of distributed neuronal activity, resulting in slower, weaker, or even absent responses to stimuli. Some studies have made similar observations in elderly subjects (e.g., [187]), which supports the idea that MCI and AD may be viewed as intensified forms of aging. 
A large palette of different stimuli and tasks are available to the EEG experimentalist; moreover, the induced responses may be analyzed in various ways, e.g., one may quantify amplitude, latency, coherence, and complexity. Some of those options have already been explored to characterize MCI and AD, as we outlined in Section 7.4.2 to 7.4.5. The results from those studies seem to be very promising: EP and ERP may complement resting EEG as diagnostic tools for MCI and AD.

In the light of the current results, it is likely that a combination of several $\mathrm{EP}$ and/or ERPs (potentially in conjunction with resting EEG) may be highly discriminative for MCI, AD, and other brain disorders. However, too few studies have so far been carried out to be able to identify the most promising approaches. Additional studies are required to further explore different types of EP and/or ERPs and to identify which of those are the most discriminative. However, we speculate that ERPs involving cognitive functions (working memory, emotions, task planning, etc.) may prove to be effective, since AD mostly induces cognitive impairments.

\subsection{Combining EEG with Other Approaches}

Virtually none of the existing EEG based methods for diagnosing AD incorporate biophysical knowledge about AD (but see [197]); detailed mathematical models of the pathology of $\mathrm{AD}$, in conjunction with EEG data analysis, may help us to improve the diagnosis of $\mathrm{AD}$ and to gain insight in the pathology of AD. Along the same lines, one may combine EEG with other imaging modalities, such as magnetic resonance imaging (MRI; e.g., [108, 157, 160, 198, 199, 200]), diffusion tensor imaging (DTI) [201], Doppler techniques [176], transcranial magnetic stimulation (TMS) [202], and single photon emission computed tomography (SPECT) [203]. We briefly outline the results from those preliminary studies.

\subsubsection{EEG and MRI}

MRI provides useful information about the anatomy of the brain; it can for example be used to measure the volume of specific brain areas, such as the hippocampus. It is well known that MCI and AD are associated with brain atrophy, most prominently in particular brain areas such as hippocampus. Brain atrophy can be detected from MRI images, by computing the volume of brain areas. It is natural to hypothesize that AD induced brain atrophy and perturbations in brain rhythms are correlated. In principle, this question may be addressed by simultaneously analyzing EEG and MRI data. Several such studies have been conducted in MCI and/or AD patients.

In [157] it was observed that the power of certain alpha sources was linearly and non-linearly correlated with the normalized hippocampal volume in MCI and AD patients; moreover, the normalized hippocampal volume was statistically greater in MCI than in AD subjects. Those results suggest that progressive atrophy of hippocampus correlates with decreased cortical alpha power, in the 
continuum along MCI and $\mathrm{AD}$ conditions. Along the same lines, a negative correlation was observed between the frontal white matter and the amplitude of frontal delta sources $(2-4 \mathrm{~Hz})$ across MCI and AD subjects [160].

In a similar study [198], it was found that increase of EEG theta/gamma and alpha3/alpha2 ratio in MCI patients can be associated with amygdalar and hippocampal atrophy respectively; moreover, amygdalo-hippocampal atrophy was seen to be associated with memory deficits. Along the same lines, a negative correlation was observed between the volume of frontal white matter and the amplitude of frontal delta sources (2-4 Hz) across MCI and AD subjects [160].

Similar findings were reported in [199], for the corpus callosum. More specifically, the correlation between interhemispheric EEG synchrony and the size of the corpus callosum was considered in AD patients and healthy subjects. The following observations were made: (i) the cross sectional area of corpus callosum was significantly reduced in AD patients relative to controls; (ii) the interhemispheric EEG coherence correlated significantly with the size of corpus callosum area. The decline in interhemispheric connectivity in $\mathrm{AD}$ may therefore be associated with a loss of cortical neurons projecting through the corpus callosum.

MRI may also be used to detect lesions in brain tissue. In particular, lesions in the cholinergic system seem to play an important role in the development of MCI and AD. Therefore, it is not improbable that such lesions are associated with the abnormal brain rhythms observed in MCI and AD patients; this issue may be addressed by combining EEG analysis with MRI. Such study was carried out in [108] for amnesic MCI (aMCI) patients; the results are as follows: the power of specific alpha and theta sources was maximum in the control subjects, intermediate in aMCI patients with little cholinergic damage, and low in aMCI patients with significant cholinergic damage. These results are consistent with the hypothesis that damage to the cholinergic system is associated with perturbations in EEG rhythms in MCI patients.

In a related study [200], the relation between vascular lesions and abnormal EEG synchrony in MCI patients was investigated; EEG synchrony (computed by a Granger measure) was highest in control subjects, intermediate in MCI patients with more vascular lesions, and low in MCI patients less vascular lesions. The results of this study seem to suggest that loss in EEG synchrony in MCI patients is not necessarily associated with cerebrovascular lesions: other factor may play a role such as neurodegenerative lesions [200].

It is evident that all those preliminary results need to be verified through numerous additional investigations. One thing is for sure though: the combination of MRI and EEG is a promising approach to relate EEG markers to brain anatomy.

\subsubsection{EEG and DTI}

Diffusion tensor imaging (DTI) is an emerging non-invasive technology to visualize subcortical fiber tracts. As we pointed out earlier, by applying synchrony measures to EEG recordings, one may assess the functional connectivity of the brain. By combining EEG and DTI, it is in principle possible to investigate 
the relation between anatomical and functional connectivity. In [201] the interhemispheric connectivity of MCI patients was studied through such combined approach; it was found that coherence in the alpha band was significantly correlated with diffusivity in particular brain areas.

\subsubsection{EEG and Doppler Techniques}

Doppler techniques allow us to visualize the cerebral blood flow; in conjunction with EEG, Doppler techniques can be used to investigate the coupling between neural activity and blood flow, often referred to as activation-flow coupling. Such study was conducted in [176], with the aim of differentiating AD patients from vascular dementia patients. Evoked EEG and blood flow responses during a visual stimulation task were analyzed; it was observed that the activation flow differs significantly between $\mathrm{AD}$ and vascular dementia patients. Therefore, simultaneous assessment of electrical as well as vascular integrity might help in differentiating both forms of dementia.

\subsubsection{EEG and TMS}

TMS can be used to evoke electrical responses in the brain. In [202] TMSevoked EEG responses in MCI and AD patients and healthy control subjects are analyzed. Significantly reduced TMS-evoked N100 and P30 was observed in the MCI and AD subjects respectively; this observation suggests that TMS may provide a novel tool for examining the degree and progression of dementia.

\subsubsection{EEG and SPECT}

SPECT may be used to visualize the metabolism and blood flow in the brain; combined with EEG, SPECT enables us to verify whether neural impairment correlate with changes in cerebral metabolism and blood flow. In [203] EEG and SPECT recordings of AD patients were simultaneously analyzed. It was found that a specific EEG measure, i.e., resting state EEG alpha dipolarity, correlates with regional cerebral blood flow; both measures seem to decline with increasing dementia severity, and they allow to some extent to separate AD patients from healthy subjects.

\subsubsection{Combining EEG with Other Approaches: Discussion}

Multi-modal approaches seem to have strong potential to improve the diagnosis of $\mathrm{AD}$ and other dementias, and many interesting topics in this area remain unexplored. The preliminary results discussed in Section 7.5.1 to 7.5.5 are just the tip of the iceberg.

First, multi-modal approaches may help us to relate characteristics of EEG in MCI and AD to neurological, vascular, or anatomical abnormalities. Second, EEG responses evoked by TMS or alternative stimulation methods that directly interact with the brain may contain more signatures of MCI and AD than standard ERPs, evoked by visual, tactile, or auditory stimulation. 


\subsection{EEG and Animal Models of AD}

In animal models of $\mathrm{AD}$, the progression of $\mathrm{AD}$ can be rigorously assessed and controlled; analyzing EEG of AD animal models may help us to relate EEG abnormalities to the neuropathology of AD. Few studies have been conducted in this important area (see, e.g., [204]).

\subsection{Effect of AD Therapies and Medication on EEG}

Another interesting topic of research is the effect of medication and therapy on the EEG of AD patients. Recent reports investigate the effect of ibuprofen [158], rivastigmine [205], cholinesterase inhibitors [206], tetrahydroaminoacridine [207], and donepezil $[159,208]$ on the EEG of AD patients. Although those studies provide exciting preliminary results, more work along those lines will be needed before any solid conclusions can be drawn.

\subsection{EEG and Risk Factors for AD}

It is unclear how risk factors for AD correlate with EEG markers. Very few studies have dealt with that topic; in [209] the relation between high plasma concentration of homocysteine and EEG abnormalities in AD patients is studied. Understanding of the interaction between AD risk factors and EEG abnormalities may lead to improved diagnostics for AD.

\section{Conclusions}

Diagnosis of AD is an important clinical problem. Since EEG is a non-invasive, simple, relatively inexpensive, and potentially mobile brain imaging technology with high temporal resolution, it seems to be a natural candidate as diagnostic tool for AD. Numerous studies indeed confirm the great potential of EEG for diagnosing $\mathrm{AD}$; moreover, some studies show promising results for MCI ("predementia"), which is the stage before AD. However, many crucial issues will need to be addressed before EEG can enter clinical practice for diagnosing AD. In other words, this research field still offers ample opportunities for exciting and clinically relevant research. We believe that this review may help to identify important research topics, which in turn may in the long term improve the reliability of EEG as a diagnostic tool for AD.

\section{Acknowledgements}

The authors wish to thank the anonymous referees for their constructive comments, which lead to many improvements to the manuscript. 


\section{References}

[1] Mattson M., 2004. Pathways towards and away from Alzheimer's disease. Nature vol. 430 .

[2] Meek P.D., McKeithan K., and Shumock G.T., 1998. Economics considerations of Alzheimer's disease. Pharmacotherapy 18(2 Pt 2):68-73.

[3] Shimokawa A., Yatomib N., Anamizuc S., Toriid S., Isonod H., Sugaid Y., and Kohnoe M., 2001. Influence of deteriorating ability of emotional comprehension on interpersonal behavior in Alzheimer-type dementia. Brain and Cognition 47(3): 423-433.

[4] Brookmeyer R., Johnson E., Ziegler-Graham K., and Arrighi M.H., 2007. Forecasting the global burden of Alzheimer's disease. Alzheimer's and Dementia 3 (3): 186-91.

[5] Cummings J.L., Frank J.C., Cherry D., Kohatsu N.D., Kemp B., Hewett L., and Mittman B., 2002. Guidelines for managing Alzheimer's disease: Part I. Assessment. American Family Physician 65(11): 2263-2272. PMID 12074525.

[6] Cummings J.L., Frank J.C., Cherry D., Kohatsu N.D., Kemp B., Hewett L., and Mittman B., 2002. Guidelines for managing Alzheimer's disease: Part II. Treatment. American Family Physician 65(12): 2525-2534. PMID 12086242.

[7] Sink K.M., Holden K.F., and Yaffe K., 2005. Pharmacological treatment of neuropsychiatric symptoms of dementia: a review of the evidence. JAMA 293 (5): 596-60.

[8] Ballard C., Lana M.M., Theodoulou M. et al, 2008. A Randomised, Blinded, Placebo-Controlled Trial in Dementia Patients Continuing or Stopping Neuroleptics (The DART-AD Trial). PLoS Med. 5 (4), 2008.

[9] Small B.J., Gagnon E., and Robinson B., 2007. Early identification of cognitive deficits: preclinical Alzheimer's disease and mild cognitive impairment. Geriatrics 62 (4): 19-23, 2007.

[10] Palmer K., Berger A.K., Monastero R., Winblad B., Bäckman L., and Fratiglioni L., 2007. Predictors of progression from mild cognitive impairment to Alzheimer disease. Neurology 68(19): 1596-1602.

[11] Arnaiz E. and Almkvist O., 2003. Neuropsychological features of mild cognitive impairment and preclinical Alzheimer's disease. Acta Neurol. Scand., Suppl. 179: 34-41, 2003.

[12] Weiner W.M., 2009. Editorial: Imaging and Biomarkers Will be Used for Detection and Monitoring Progression of Early Alzheimer's Disease. J. Nutr. Health Aging 4:332.

[13] Sunderland T., Hampel H., Takeda M., Putnam K.T., and Cohen R.M., 2006. Biomarkers in the diagnosis of Alzheimer's disease: are we ready? J Geriatr Psychiatry Neurol. 19(3):172-9.

[14] Jeong J., 2004. EEG Dynamics in patients with Alzheimer's disease. Clinical Neurophysiology 115, 1490-1505.

[15] Jackson C.E. and Snyder P.J., 2008. Electroencephalography and event-related potentials as biomarkers of mild cognitive impairment and mild Alzheimer's disease. Alzheimers Dement. 2008 4(1 Suppl 1):137-43. 
[16] Prichep LS., 2007. Quantitative EEG and electromagnetic brain imaging in aging and in the evolution of dementia. Ann NY Acad Sci. 1097:156-67.

[17] Adeli H., Ghosh-Dastidar S., and Dadmehr N., 2005. Alzheimer's disease: models of computation and analysis of EEGs. Clin EEG Neurosci. 36(3):131-40.

[18] Verleger R., 1991. The instruction to refrain from blinking affects auditory P3 and N1 amplitudes. Electroencephalogr Clin Neurophysiol. 78(3):240-51.

[19] Hoffmann S. and Falkenstein M., 2008. The correction of eye blink artefacts in the EEG: a comparison of two prominent methods. PLoS ONE 3(8):e3004.

[20] Dammers J., Schiek M., Boers F., Silex C., Zvyagintsev M., Pietrzyk U., and Mathiak K. , 2008. Integration of amplitude and phase statistics for complete artifact removal in independent components of neuromagnetic recordings. IEEE Trans Biomed Eng. 55(10):2353-62.

[21] Delorme A., Sejnowski T., and Makeig S., 2007. Enhanced detection of artifacts in EEG data using higher-order statistics and independent component analysis. Neuroimage 34(4):1443-9.

[22] Romero S., Mañanas M.A., and Barbanoj M.J., 2009. Ocular reduction in EEG signals based on adaptive filtering, regression and blind source separation. Ann Biomed Eng. 37(1):176-91.

[23] Mammone N. and Morabito F.C., 2008. Enhanced automatic artifact detection based on independent component analysis and Renyi's entropy. Neural Netw. 21(7):1029-40.

[24] Goncharova I.I., McFarland D.J., Vaughan T.M., and Wolpaw J.R., 2003. EMG contamination of EEG: spectral and topographical characteristics. Clin Neurophysiol. 114(9):1580-93.

[25] van Boxtel A., 2001. Optimal signal bandwidth for the recording of surface EMG activity of facial, jaw, oral, and neck muscles. Psychophysiology 38(1):22-34.

[26] van Boxtel A., Goudswaard P., and Schomaker L.R., 1984. Amplitude and bandwidth of the frontalis surface EMG: effects of electrode parameters. Psychophysiology 21(6):699-707.

[27] van Boxtel A., Goudswaard P., van der Molen G.M., and van den Bosch W.E., 1983. Changes in electromyogram power spectra of facial and jaw-elevator muscles during fatigue. J Appl Physiol. 54(1):51-8.

[28] Barlow, J.S., 1986. Artifact processing (rejection and minimization) in EEG data processing. In: F.H. Lopes da Silva, W. Storm van Leeuwen and A. Remond (Eds.). Handbook of electroencephalography and clinical neurophysiology, volume 2. Revised series. Amsterdam: Elsevier, 15-62.

[29] Crespo-Garcia M., Atienza M., and Cantero J.L., 2008. Muscle artifact removal from human sleep EEG by using independent component analysis. Ann Biomed Eng. 36(3):467-75.

[30] P. Nunez and R. Srinivasan, Electric Fields of the Brain: The Neurophysics of EEG, Oxford University Press, 2006.

[31] Celka P., Boashash B., and Colditz P., 2001. Preprocessing and time-frequency analysis of newborn EEG seizures. IEEE Eng Med Biol Mag. 20(5):30-9.

[32] Bonmassar G., Purdon P.L., Jääskeläinen I.P., Chiappa K., Solo V., Brown E.N., and Belliveau J.W., 2002. Motion and ballistocardiogram artifact removal for interleaved recording of EEG and EPs during MRI. Neuroimage 16(4):1127-41. 
[33] He P., Wilson G., and Russell C., 2004. Removal of ocular artifacts from electroencephalogram by adaptive filtering. Med Biol Eng Comput. 42(3):407-12.

[34] Schlögl A., Anderer P., Roberts S.J., Pregenzer M., and Pfurtscheller G., 1999. Artefact detection in sleep by the use of Kalman filtering. Proc EMBC 1999, Part II, $1648-1649$.

[35] Kierkels J.J., Riani J., Bergmans J.W., and van Boxtel G.J., 2007. Using an eye tracker for accurate eye movement artifact correction. IEEE Trans Biomed Eng. $54(7): 1256-67$.

[36] Croft R.J. and Barry R.J. Removal of ocular artifact from the EEG: a review. Neurophysiol Clin. 30(1):5-19.

[37] Moretti D.V., Babiloni F., Carducci F., Cincotti F., Remondini E., Rossini P.M., Salinari S., and Babiloni C., 2003. Computerized processing of EEG-EOG-EMG artifacts for multi-centric studies in EEG oscillations and event-related potentials. Int J Psychophysiol. 47(3):199-216.

[38] He P., Wilson G., Russell C., and Gerschutz M., 2007. Removal of ocular artifacts from the EEG: a comparison between time-domain regression method and adaptive filtering method using simulated data. Med Biol Eng Comput. 45(5):495-503.

[39] Schlögl A., Keinrath C., Zimmermann D., Scherer R., Leeb R., and Pfurtscheller G. A fully automated correction method of EOG artifacts in EEG recordings. Clin Neurophysiol. 118(1):98-104.

[40] Cichocki A. and Amari S.I., 2002. Adaptive Blind Signal and Image Processing: Learning Algorithms and Applications. Wiley, New York.

[41] Jung T.P., Makeig S., Humphries C., Lee T.W., McKeown M.J., Iragui V., and Sejnowski T.J., 2000. Removing electroencephalographic artifacts by blind source separation. Psychophysiology 37(2):163-78.

[42] Jung T.P., Makeig S., Westerfield M., Townsend J., Courchesne E., and Sejnowski T.J., 2000. Removal of eye activity artifacts from visual event-related potentials in normal and clinical subjects. Clin Neurophysiol. 111(10):1745-58.

[43] Vialatte F.B., Solé-Casals J., and Cichocki A., 2007. EEG windowed statistical wavelet deviation for estimation of muscular artifacts. Proc. IEEE 32nd Int. Conf. on Acoustics, Speech, and Signal Processing (ICASSP'07) vol. 4, 1161-4.

[44] Vialatte F.B., Solé-Casals J., and Cichocki A. EEG windowed statistical wavelet scoring for evaluation and discrimination of muscular artifacts. Physiol Meas. 29(12):1435-52.

[45] Cichocki A., Shishkin S.L., Musha T., Leonowicz Z., Asada T., and Kurachi T., 2005. EEG filtering based on blind source separation (BSS) for early detection of Alzheimer's disease. Clin Neurophysiol. 116(3):729-37.

[46] Melissant C., Ypma A., Frietman E.E., and Stam C.J., 2005. A method for detection of Alzheimer's disease using ICA-enhanced EEG measurements. Artif Intell Med. 33(3):209-22.

[47] Solé-Casals J., Vialatte F., Haenschel C., Pantel J., Prvulovic D., and Cichocki A., 2009. Improving early detection of Alzheimers disease using ICA cleaning procedures. Proc. Neuromath 2009. 
[48] Solé-Casals J., Vialatte F.B., Chen Z., and Cichocki A., 2009. Coherency and sharpness measures by using ICA algorithms. An investigation for Alzheimers disease discrimination. 2nd International Conference on Bio-inspired systems and signal processing, Porto, Portugal, January 14-17 2009. Proc. Biosignals 2009.

[49] Solé-Casals J., Vialatte F.B., Chen Z., and Cichocki A., 2008. Investigation of ICA algorithms for feature extraction of EEG signals in discrimination of Alzheimer disease. 1st International Conference on Bio-inspired systems and signal processing, Funchal, Madeira, Portugal, January 28-31 2008. Proc. Biosignals 2008.

[50] Vialatte F.B., Solé-Casals J., Maurice M., Latchoumane C., Hudson N., Wimalaratna S., Jeong J., and Cichocki A., 2008. Improving the Quality of EEG Data in Patients With Alzheimers Disease Using ICA. Proc. 15th International Conference on Neural Information Processing, ICONIP 2008, LNCS, Part II, 5507:979986.

[51] Vialatte F.B., Bakardjian H., Prasad R., and Cichocki A. EEG paroxysmal gamma waves during Bhramari Pranayama: A yoga breathing technique. Conscious Cogn., in press.

[52] Baker M., Akrofi K., Schiffer R., and Michael W. O’ Boyle, 2008. EEG Patterns in Mild Cognitive Impairment (MCI) Patients. Open Neuroimag J. 2: 52-55.

[53] Besthorn C., Zerfass R., Geiger-Kabisch C., Sattel H., Daniel S., Schreiter-Gasser U., and Frstl H., 1997. Discrimination of Alzheimer's disease and normal aging by EEG data. Electroencephalography and Clinical Neurophysiology 103:241-248.

[54] van der Hiele K., Vein A.A., Reijntjes R.H., Westendorp R.G., Bollen E.L., van Buchem M.A., van Dijk J.G., Middelkoop H.A., 2007. EEG correlates in the spectrum of cognitive decline. Clin Neurophysiol. 118(9):1931-9.

[55] Czigler B., Csikós D., Hidasi Z., Anna Gaál Z., Csibri E., Kiss E., Salacz P., Molnár M., 2008. Quantitative EEG in early Alzheimer's disease patients-power spectrum and complexity features. Int. J. Psychophysiol. 68(1):75-80.

[56] Gianotti L.R., Künig G., Lehmann D., Faber P.L., Pascual-Marqui R.D., Kochi K., and Schreiter-Gasser U., 2007. Correlation between disease severity and brain electric LORETA tomography in Alzheimer's disease. Clin. Neurophysiol. 118(1):186-96.

[57] Herrmann C.S. and Demiralp T., 2005. Human EEG gamma oscillations in neuropsychiatric disorders. Clin. Neurophysiol. 116(12):2719-33.

[58] Latchoumane C.F.V., Vialatte F.B., Cichocki A., and Jeong J. Multiway analysis of Alzheimers disease: classification based on space-frequency characteristics of EEG time series, 2008. Proc. World Congress on Engineering 2008, London, United Kingdom, 2-4 July.

[59] Latchoumane C.F.V, Vialatte F.B., Jeong J., and Cichocki A. EEC Classification of Mild and Severe Alzheimer's Disease using Parallel Factor Analysis Method, 2009. In Advances in Electrical Engineering and Computational Science, in press.

[60] Schreiter Gasser U., Rousson V., Hentschel F., Sattel H., Gasser T., 2008. Alzheimer disease versus mixed dementias: an EEG perspective. Clin Neurophysiol 119(10):2255-9.

[61] Moretti D.V., Fracassi C., Pievani M., Geroldi C., Binetti G., Zanetti O., Sosta K., Rossini P.M., and Frisoni G.B., 2009. Increase of theta/gamma ratio is associated with memory impairment. Clin. Neurophysiol. 120(2):295-303. 
[62] Polikar R., Topalis A., Green D., Kounios J., and Clark C.M., 2007. Comparative multiresolution wavelet analysis of ERP spectral bands using an ensemble of classifiers approach for early diagnosis of Alzheimer's disease. Comput Biol Med. 37(4):542-58.

[63] Vialatte F.B., Maurice M., and Cichocki A., 2008. Why sparse bump models? OHBM meeting, Melbourne, Australia, June 15-19 2008. Neuroimage, 41(S1):S159.

[64] Vialatte F.B., Sole-Casals J., Hazart A., Prvulovic D., Dauwels J., Pantel J., and Haenschel C., 2009. Modeling transient oscillations in the EEG of patients with mild cognitive impairment. Proc. 2nd International Conference on Cognitive Neurodynamics (ICCN09).

[65] Vialatte F., Cichocki A., Dreyfus G., Musha T., Shishkin S.L., and Gervais R., 2005. Early Diagnosis of Alzheimer's Disease by Blind Source Separation, Time Frequency Representation, and Bump Modeling of EEG Signals. Proc. 15th International Conference on Artificial Neural Networks (Part I, Eds. W. Duch, J. Kacprzyk, E. Oja, and S. Zadrozny,), Warsaw, Poland, September 11-15. Springer, LNCS 3696, pp. 683-692.

[66] Barlow J. S., The Electroencephalogram: Its Patterns and Origins, MIT Press, 1993.

[67] van Deursen J.A., Vuurman E.F., Verhey F.R., van Kranen-Mastenbroek V.H., and Riedel W.J., 2009. Increased EEG gamma band activity in Alzheimer's disease and mild cognitive impairment. J Neural Transm. 115(9):1301-11.

[68] Vialatte F., 2005. Modélisation en bosses pour l'analyse des motifs oscillatoires reproductibles dans l'activité de populations neuronales: applications à l'apprentissage olfactif chez l'animal et à la détection précoce de la maladie d'Alzheimer, $\mathrm{PhD}$ Thesis, Paris VI University, Paris.

[69] Vialatte F., Martin C., Dubois R., Haddad J., Quenet B., Gervais R., and Dreyfus G., 2007. A machine learning approach to the analysis of time-frequency maps, and its application to neural dynamics. Neural Networks 20, 194-209.

[70] Vialatte F., J. Sol-Casals, J. Dauwels, M. Maurice, and A. Cichocki, 2009. Bump time-frequency toolbox: a toolbox for time-frequency oscillatory bursts extraction in electrophysiological signals. BMC Neuroscience 10:46.

[71] Shannon C.E., 1948. A Mathematical Theory of Communication. Bell System Technical Journal 27:379-423, 623-656.

[72] Cover T. M. and Thomas J. A., 1991. Elements of Information Theory. Wiley, New York.

[73] Pincus S.M., 1991. Approximate entropy as a measure of system complexity. Proc Natl Acad Sci USA 88:2297-2301.

[74] Lake D.E., Richman J.S, Griffin M.P., and Moorman J.R., 2002. Sample entropy analysis of neonatal heart rate variability. Am J Physiol 283(3):R789-R797.

[75] Costa M., Goldberger A.L., Peng C.-K., 2002. Multiscale entropy analysis of physiologic time series. Phys Rev Lett 89:062102.

[76] Costa M., Goldberger A.L., and Peng C.-K., 2005. Multiscale entropy analysis of biological signals. Phys Rev E 71:021906.

[77] Jeong J., Gore J., and Peterson B., 2001. Mutual information analysis of the EEG in patients with Alzheimer's disease. Clinical Neurophysiology 112, 827-835. 
[78] Lempel A. and Ziv J., 1976. On the complexity of finite sequences. IEEE Transactions on Information Theory 22:75-81.

[79] Ziv J. and Lempel A., 1978. A universal algorithm for sequential data compression. IEEE Transactions on Information Theory 23:337-343.

[80] Welch T.A., 1984. A technique for high-performance data compression. IEEE Computer 17:8-19.

[81] Ziv J. and Lempel A., 1978. Compression of individual sequences via variable-rate coding. IEEE Transactions on Information Theory 24:530-536.

[82] Zhao P., Van-Eetvelt P., Goh C., Hudson N., Wimalaratna S., and Ifeachor E., 2007. Characterization of EEGs in Alzheimer's disease using information theoretic methods. Conf Proc 2007 IEEE Eng Med Biol Soc. pp. 5127-31.

[83] Mandelbrot B.B., 1982. The Fractal Geometry of Nature. W.H. Freeman and Company.

[84] Grassberger P., 1983. Generalized dimensions of strange attractors. Physics Letters A 97 (6):227-230.

[85] Kaplan J. and Yorke J., 1987. Chaotic behavior of multidimensional difference equations. In Peitgen, H.O. \& Walther, H.O., editors, Functional Differential Equations and Approximation of Fixed Points Springer, New York.

[86] Pesin Y.B., 1977. Characteristic Lyapunov exponents and smooth ergodic theory. Russian Math. Surveys 32(4):55-114.

[87] Hornero R., Abásolo D., Escudero J., and Gómez C., 2009. Nonlinear analysis of electroencephalogram and magnetoencephalogram recordings in patients with Alzheimer's disease. Phil. Trans. R. Soc. A 367:317-336.

[88] Abásolo D., Escudero J., Hornero R., Gómez C., and Espino P., 2008. Approximate entropy and auto mutual information analysis of the electroencephalogram in Alzheimer's disease patients. Med. Biol. Eng. Comput. (10):1019-28.

[89] Abásolo D., Hornero R., Espino P., Alvarez D., and Poza J., 2006. Entropy analysis of the EEG background activity in Alzheimer's disease patients. Physiol Meas. $27(3): 241-53$.

[90] Abásolo D., Hornero R., Espino P., Poza J., Sánchez C.I., and de la Rosa R., 2005. Analysis of regularity in the EEG background activity of Alzheimer's disease patients with Approximate Entropy. Clin Neurophysiol. 2005 Aug;116(8):1826-34.

[91] Besthorn C., Sattel H., Geiger-Kabisch C., Zerfass R. and Förstl H., 1995. Parameters of EEG dimensional complexity in Alzheimer's disease. Electroencephalography and Clinical Neurophysiology 95:8489.

[92] B. Jelles, J.H. van Birgelen, J.P.J. Slaets, R.E.M. Hekster, E.J. Jonkman and C.J. Stam, 1999. Decrease of non-linear structure in the EEG of Alzheimer's patients compared to healthy controls. Clinical Neurophysiology 110:1159-1167.

[93] Adeli H., Ghosh-Dastidar S., Dadmehr N., 2008. A spatio-temporal waveletchaos methodology for EEG-based diagnosis of Alzheimer's disease. Neurosci Lett. 444(2):190-4.

[94] Woon W., Cichocki A., Vialatte F., and Musha T., 2007. Techniques for early detection of Alzheimer's disease using spontaneous EEG recordings. Physiol. Meas. 28 335-347. 
[95] Park J-H., Kim S., Kim C-H., Cichocki A., and Kim K., 2007. Multiscale Entropy Analysis of EEG from Patients under Different Pathological Conditions. Fractals 15(4):399-404.

[96] Dauwels J., Vialatte F., and Cichocki A., 2007. A comparative study of synchrony measures for the early detection of AD. Lecture Notes in Computer Science 4985, Springer.

[97] Dauwels J., Vialatte F., and Cichocki A., 2009. A comparative study of synchrony measures for the early diagnosis of Alzheimer's disease based on EEG. NeuroImage, in press.

[98] Dauwels J., Vialatte F., Latchoumane C., Jeong J., and Cichocki A., 2009. Loss of EEG Synchrony in Early-Stage AD patients: a Study with Multiple Synchrony Measures and Multiple EEG Data Sets. Proc. 31th Annual International Conference of the IEEE Engineering in Medicine and Biology Society (EMBC09), in press

[99] Stevens A., Kircher T., Nickola M., Bartels M., Rosellen N., and Wormstall H., 2001. Dynamic regulation of EEG power and coherence is lost early and globally in probable DAT. Eur. Arch. Psychiatry Clin. Neurosci. 251, 199-204.

[100] Brassen S., Braus D., Weber-Fahr W., Trost H., Moritz S., and Adler G., 2004. Late-onset depression with mild cognitive deficits: electrophysiological evidences for a preclinical dementia syndrome. Dementia and Geriatric Cognitive Disorders 18, $271-277$.

[101] Locatelli T., Cursi M., Liberati D., Franceschi M., and Comi G., 1998. EEG coherence in Alzheimer's disease. Electroencephalogr. Clin. Neurophysiol. 106, 22937.

[102] Wada Y., Nanbu Y., Koshino Y., Yamaguchi N., and Hashimoto T., 1998. Reduced interhemispheric EEG coherence in Alzheimer's disease: analysis during rest and photic stimulation. Alzheimer Dis. Assoc. Disord. 12, 175-81.

[103] Hogan M., Swanwicka G., Kaiserb J., Rowanc M. and Lawlora B., 2003. Memoryrelated EEG power and coherence reductions in mild Alzheimer's disease. Int. J. Psychophysiol. 49, 147-163.

[104] Anghinah R., Kanda P., Jorge M., De Lima E., Pascuzzi L., and De Paiva Melo A., 2000. Alpha band coherence analysis of EEG in healthy adult and Alzheimer's type dementia subjects. Arq. Neuropsiquiatr. 58, 272-275.

[105] Hidasi Z., Czigler B., Salacz P., Csibri E., Molnár M., 2007. Changes of EEG spectra and coherence following performance in a cognitive task in Alzheimer's disease. Int. J. Psychophysiol. 65(3):252-260.

[106] Jelles B., Scheltens P., van der Flier W.M., Jonkman E.J., da Silva F.H., and Stam C.J., 2008. Global dynamical analysis of the EEG in Alzheimer's disease: frequency-specific changes of functional interactions. Clin. Neurophysiol. 119(4):837-841.

[107] Stam C.J., Nolte G., Daffertshofer A., 2007. Phase lag index: assessment of functional connectivity from multi channel EEG and MEG with diminished bias from common sources. Hum. Brain Mapp. 28(11):1178-1193.

[108] Babiloni C., Pievani M., Vecchio F., Geroldi C., Eusebi F., Fracassi C., Fletcher E., De Carli C., Boccardi M., Rossini P.M., Frisoni G.B., 2009. White-matter lesions along the cholinergic tracts are related to cortical sources of EEG rhythms in amnesic mild cognitive impairment. Hum. Brain Mapp. 30(5):1431-43. 
[109] Babiloni C., Ferri R., Binetti G., Vecchio F., Frisoni G.B., Lanuzza B., Miniussi C., Nobili F., Rodriguez G., Rundo F., Cassarino A., Infarinato F., Cassetta E., Salinari S., Eusebi F., Rossini P.M., 2009. Directionality of EEG synchronization in Alzheimer's disease subjects. Neurobiol Aging 30(1):93-102.

[110] Kramer M., Chang F. L., Cohen M., Hudson D., and Szeri A., 2007. Synchronization measures of the scalp EEG can discriminate healthy from Alzheimer's subjects. International Journal of Neural Systems 17, 1-9.

[111] Stam C. J., van der Made Y., Pijnenburg Y. A. L., and Scheltens Ph., 2003. EEG synchronization in mild cognitive impairment and Alzheimer's disease. Acta Neurologica Scandinavica 108, 90-96.

[112] Stam C. J., Montez T., Jones B. F., Rombouts S. A. R. B., van der Made Y., Pijnenburg Y. A. L., and Scheltens Ph., 2005. Disturbed fluctuations of resting state EEG synchronization in Alzheimer's disease. Clinical Neurophysiology 116, 708-715.

[113] Babiloni C., Ferri R., Binetti G., Cassarino A., Forno G. D., Ercolani M., Ferreri F., Frisoni G. B., Lanuzza B., Miniussi C., Nobili F., Rodriguez G., Rundo F., Stam C. J., Musha T., Vecchio F., and Rossini P. M., 2006. Fronto-parietal coupling of brain rhythms in mild cognitive impairment: a multicentric EEG study. Brain Res. Bull. 69, 63-73.

[114] Pijnenburg Y. A., van de Made Y., van Cappellen van Walsum A. M., Knol D. L., Scheltens P., Stam C. J., 2004. EEG Synchronization likelihood in mild cognitive impairment and Alzheimer's disease during a working memory task. Clin. Neurophysiol. 115, 1332-9.

[115] Wan B., Ming D., Qi H., Xue Z., Yin Y., Zhou Z., Cheng L., 2008. Linear and nonlinear quantitative EEG analysis. IEEE Eng. Med. Biol. Mag. 27(5):58-63.

[116] Pijnenburg Y.A., Strijers R.L., Made Y.V., van der Flier W.M., Scheltens P., and Stam C.J., 2008. Investigation of resting-state EEG functional connectivity in frontotemporal lobar degeneration. Clin Neurophysiol. 119(8):1732-8.

[117] Koenig T., Prichep L., Dierks T., Hubl D., Wahlund L. O., John E. R., and Jelic V., 2005. Decreased EEG synchronization in Alzheimer's disease and mild cognitive impairment. Neurobiol. Aging. 26, 165-71.

[118] Park Y.M., Che H.J., Im C.H., Jung H.T., Bae S.M., and Lee S.H., 2008. Decreased EEG synchronization and its correlation with symptom severity in Alzheimer's disease. Neurosci Res. 62(2):112-7.

[119] Dauwels J., Vialatte F., Rutkowski T., and Cichocki A., 2007. Measuring neural synchrony by message passing, Advances in Neural Information Processing Systems 20 (NIPS 20), in press.

[120] Dauwels, J., F. Vialatte, T. Weber, and A. Cichocki, 2009. Quantifying statistical interdependence by message passing on graphs, Part I: One-dimensional point processes. Neural Computation 21:8, in press.

[121] Dauwels J., F. Vialatte, T. Weber, T. Musha, and A. Cichocki, 2009. Quantifying statistical interdependence by message passing on graphs, Part II: Multi-dimensional point processes. Neural Computation 21:8, in press.

[122] Dauwels J., Weber T., Vialatte F.B., and Cichocki A., 2008. Quantifying the Similarity of Multiple Multi-Dimensional Point Processes by Integer Programming 
with Application to Early diagnosis of Alzheimers Disease from EEG. Proc. 30th IEEE International Conference of the Engineering in Medicine and Biology Society, EMBC, August 20-24, 2008, Vancouver, BC, Canada.

[123] Stam C. J., 2005. Nonlinear dynamical analysis of EEG and MEG: review of an emerging field. Clinical Neurophysiology 116, 2266-2301.

[124] Quiroga R. Q., Kraskov A., Kreuz T., and Grassberger P., 2002. Performance of different synchronization measures in real data: a case study on EEG signals. Physical Review E 65.

[125] Pereda E., Quiroga R. Q., and Bhattacharya J., 2005. Nonlinear multivariate analysis of neurophsyiological signals. Progress in Neurobiology 77, 1-37.

[126] Gunduz A. and J.C. Principe J.C., 2009. Correntropy as a novel measure for nonlinearity tests. Signal Processing 89(1): 14-23.

[127] Kamiński M. and Hualou Liang, 2005. Causal influence: advances in neurosignal analysis. Critical review in biomedical engineering 33, (4):347-430.

[128] Ancona N., Marinazzo D., and Stramaglia S., 2004. Radial basis function approach to nonlinear Granger causality of time series. Physical Review E 70.

[129] Chen Y., Rangarajan G., Feng J., and Ding M., 2004. Analyzing multiple nonlinear time series with extended Granger causality. Physics Letters A 324, 26-35.

[130] Lachaux J.-P., Rodriguez E., Martinerie J., and Varela F. J., 1999. Measuring phase synchrony in brain signals. Human Brain Mapping 8, 194-208.

[131] Nolte G., Wheaton O.B.L., Mari Z., Vorbach S., and Hallett M., 2004. Identifying true brain interaction from EEG data using the imaginary part of coherency. Clin Neurophysiol 115:2292-2307.

[132] Koenig T., Lehmann D., Saito N., Kuginuki T., Kinoshita T., and Koukkou M., 2001. Decreased functional connectivity of EEG theta-frequency activity in first-episode, neuroleptic-naive patients with schizophrenia: preliminary results. Schizophrenia Res. 1-2, 55-60.

[133] Stam C. J. and van Dijk B. W., 2002. Synchronization likelihood: an unbiased measure of generalized synchronization in multivariate data sets. Physica D 163, $236-251$.

[134] Saito N., Kuginuki T., Yagyu T., Kinoshita T., Koenig T., Pascual-Marqui R. D., Kochi K., Wackermann J., and Lemann D., 1998. Global, regional and local measures of complexity of multichannel EEG in acute, neuroleptic-naive, first-break schizophrenics. Society of Biological Psychiatry. 43:794-802.

[135] Carmeli C., Knyazeva M. G., Innocenti G. M., and De Feo O., 2005. Assessment of EEG synchronization based on state-space analysis. Neuroimage 25, 339-354.

[136] Kraskov A., Stögbauer H., and Grassberger P., 2004. Estimating mutual information. Phys. Rev. E 69.

[137] Aviyente S., 2005. A measure of mutual information on the time-frequency plane. Proc. of ICASSP 2005, 481-484.

[138] Aviyente S., 2005. Information-theoretic signal processing on the time-frequency plane and applications. Proc. of EUSIPCO 2005.

[139] Blanco S., Quiroga R. Q., Rosso O., and Kochen S., 1995. Time-frequency analysis of EEG series. Physical Review E 51. 
[140] Quiroga Q. R., Rosso O., and Basar E., 1999. Wavelet-entropy: a measure of order in evoked potentials. Electr. Clin. Neurophysiol. (Suppl.) 49, 298-302.

[141] Besthorn C., Forstl H., Geiger-Kabisch C., Sattel H., Gasser T., and SchreiterGasser U, 1994. EEG Coherence in Alzheimer's disease. Electroencephalogr Clin Neurophysiol 90, 242-5.

[142] Güntekin B, Saatçi E, Yener G., 2009. Decrease of evoked delta, theta and alpha coherences in Alzheimer patients during a visual oddball paradigm. Brain Res. 1235:109-16.

[143] Jiang Z. Y., 2005. Abnormal Corticol Functional Connections in Alzheimer's Disease: Analysis of Inter- and Intra-Hemispheric EEG Coherence. J Zhejiang Univ Sci B. 6, 259-264.

[144] Knyazeva M.G., Jalili M., Brioschi A., Bourquin I., Fornari E., Hasler M., Meuli R., Maeder P., Ghika J., 2008. Topography of EEG multivariate phase synchronization in early Alzheimer's disease. Neurobiol Aging.

[145] Yagyu T., Wackermann J., Shigeta M., Jelic V., Kinoshita T., K. Kochi, P. Julin, O. Almkvist, L. O. Wahlund, I. Kondakor, and Lehmann D., 1997. Global dimensional complexity of multichannel EEG in mild Alzheimer's disease and age-matched cohorts. Dement. Geriatr. Cogn. Disord. 8, 343-7.

[146] Yoshimura M., Isotani T., Yagyu T., Irisawa S., Yoshida T., Sugiyama M., Minami T., Sugimoto T., Nobuhara K., Okugawa G., and Kinoshita T., 2004. Global approach to multichannel electroencephalogram analysis for diagnosis and clinical evaluation in mild Alzheimer's disease. Neuropsychobiology 49:163-166.

[147] Watts D.J. and Strogatz S.H., 1998. Collective dynamics of "smallworld" networks. Nature 393(6684):440-2.

[148] Stam C.J., Jones B.F., Nolte G., Breakspear M., Scheltens P., 2007. Smallworld networks and functional connectivity in Alzheimer's disease. Cereb Cortex 17(1):92-9.

[149] He Y., Chen Z., Gong G., Evans A., 2009. Neuronal Networks in Alzheimer's Disease. Neuroscientist.

[150] Jiang Z. Y., 2005. Study on EEG Power and Coherence in Patients with Mild Cognitive Impairment During Working Memory Task. J. Zhejiang Univ. Sci B. 6, 1213-9.

[151] Jiang Z. Y. and Zheng L. L., 2006. Inter-and intra-hemispheric EEG coherence in patients with mild cognitive impairment at rest and during working memory task. J. Zhejiang Univ Sci B. 7, 357-364.

[152] Uhlhaas P. and Singer W., 2006. Neural synchrony in brain disorders: relevance for cognitive dysfunctions and pathophysiology. Neuron 52, 155-168.

[153] Babiloni C., Visser P.J., Frisoni G., De Deyn P.P., Bresciani L., Jelic V., Nagels G., Rodriguez G., Rossini P.M., Vecchio F., Colombo D., Verhey F., Wahlund L.O., and Nobili F., 2009. Cortical sources of resting EEG rhythms in mild cognitive impairment and subjective memory complaint. Neurobiol Aging, in press.

[154] Rossini P.M., Del Percio C., Pasqualetti P., Cassetta E., Binetti G., Dal Forno G., Ferreri F., Frisoni G., Chiovenda P., Miniussi C., Parisi L., Tombini M., Vecchio F., and Babiloni C., 2006. Conversion from mild cognitive impairment to Alzheimer's disease is predicted by sources and coherence of brain electroencephalography rhythms. Neuroscience 143(3):793-803. 
[155] Babiloni C., Cassetta E., Binetti G., Tombini M., Del Percio C., Ferreri F., Ferri R., Frisoni G., Lanuzza B., Nobili F., Parisi L., Rodriguez G., Frigerio L., Gurzì M., Prestia A., Vernieri F., Eusebi F., and Rossini P.M., 2007. Resting EEG sources correlate with attentional span in mild cognitive impairment and Alzheimer's disease. Eur J Neurosci. 25(12):3742-57.

[156] Juckel G., Clotz F., Frodl T., Kawohl W., Hampel H., Pogarell O., and Hegerl U., 2008. Diagnostic usefulness of cognitive auditory event-related P300 subcomponents in patients with Alzheimers disease? J Clin Neurophysiol. 25(3):147-52.

[157] Babiloni C., Frisoni G.B., Pievani M., Vecchio F., Lizio R., Buttiglione M., Geroldi C., Fracassi C., Eusebi F., Ferri R., and Rossini P.M., 2009. Hippocampal volume and cortical sources of EEG alpha rhythms in mild cognitive impairment and Alzheimer disease. Neuroimage 44(1):123-35.

[158] Babiloni C., Frisoni G.B., Del Percio C., Zanetti O., Bonomini C., Cassetta E., Pasqualetti P., Miniussi C., De Rosas M., Valenzano A., Cibelli G., Eusebi F., Rossini P.M., 2009. Ibuprofen treatment modifies cortical sources of EEG rhythms in mild Alzheimer's disease. Clin. Neurophysiol. 120(4):709-18.

[159] Babiloni C., Cassetta E., Dal Forno G., Del Percio C., Ferreri F., Ferri R., Lanuzza B., Miniussi C., Moretti D.V., Nobili F., Pascual-Marqui R.D., Rodriguez G., Luca Romani G., Salinari S., Zanetti O., and Rossini P.M., 2006. Donepezil effects on sources of cortical rhythms in mild Alzheimer's disease: Responders vs. Non-Responders. Neuroimage 31(4):1650-65.

[160] Babiloni C., Frisoni G., Steriade M., Bresciani L., Binetti G., Del Percio C., Geroldi C., Miniussi C., Nobili F., Rodriguez G., Zappasodi F., Carfagna T., and Rossini P.M. Frontal white matter volume and delta EEG sources negatively correlate in awake subjects with mild cognitive impairment and Alzheimer's disease. Clin Neurophysiol. 117(5):1113-29.

[161] Bonferroni C. E., 1936. Teoria statistica delle classi e calcolo delle probabilitá. Pubblicazioni del R. Instituto Superiore di Scienze Economiche e Commerciali di Firenze 8, 3-62.

[162] Storey J., 2002. A direct approach to false discovery rates. Journal of the Royal Statistical Society, Series B (Methodological) 64 (3): 479-498.

[163] Lehmann C., Koenig T., Jelic V., Prichep L., John R.E., Wahlund L.O., Dodge Y., and Dierks T., 2007. Application and comparison of classification algorithms for recognition of Alzheimer's disease in electrical brain activity (EEG). J Neurosci Methods. 161(2):342-50.

[164] Buscema M, Rossini P, Babiloni C, and Grossi E., 2007. The IFAST model, a novel parallel nonlinear EEG analysis technique, distinguishes mild cognitive impairment and Alzheimer's disease patients with high degree of accuracy. Artif Intell Med. 40(2):127-41.

[165] Chapman R.M., Nowlis G.H., McCrary J.W., Chapman J.A., Sandoval T.C., Guillily M.D., Gardner M.N., and Reilly L.A., 2007. Brain event-related potentials: diagnosing early-stage Alzheimer's disease. Neurobiol Aging. 28(2):194-201.

[166] Goldberger A.L., Amaral L.A.N., Glass L., Hausdorff J.M., Ivanov P.Ch., Mark R.G., Mietus J.E., Moody G.B., Peng C.K., and Stanley H.E. PhysioBank, PhysioToolkit, and PhysioNet: Components of a New Research Resource for Complex Physiologic Signals. Circulation 101(23):e215-e220 [Circulation Electronic Pages; http://circ.ahajournals.org/cgi/content/full/101/23/e215]; 2000 (June 13). 
[167] Liddell B.J., Paul R.H., Arns M., Gordon N., Kukla M., Rowe D., Cooper N., Moyle J., and Williams L.M., 2007. Rates of decline distinguish Alzheimer's disease and mild cognitive impairment relative to normal aging: integrating cognition and brain function. J. Integr. Neurosci. 6(1):141-74.

[168] Olichney J.M., Taylor J.R., Gatherwright J., Salmon D.P., Bressler A.J., Kutas M., and Iragui-Madoz V.J., 2008. Patients with MCI and N400 or P600 abnormalities are at very high risk for conversion to dementia. Neurology 70(19 Pt 2):1763-70.

[169] Missonnier P., Deiber M.P., Gold G., Herrmann F.R., Millet P., Michon A., Fazio-Costa L., Ibañez V., and Giannakopoulos P., 2007. Working memory loadrelated electroencephalographic parameters can differentiate progressive from stable mild cognitive impairment. Neuroscience 150(2):346-56.

[170] Bennys K., Portet F., Touchon J., and Rondouin G., 2007. Diagnostic value of event-related evoked potentials N200 and P300 subcomponents in early diagnosis of Alzheimer's disease and mild cognitive impairment. J Clin Neurophysiol. 24(5):40512.

[171] Giannakopoulos P., Missonnier P., Kövari E., Gold G., and Michon A., 2009. Electrophysiological markers of rapid cognitive decline in mild cognitive impairment. Front Neurol Neurosci. 24:39-46.

[172] Missonnier P., Gold G., Fazio-Costa L., Michel J.P., Mulligan R., Michon A., Ibáñez V., Giannakopoulos P., 2005. Early event-related potential changes during working memory activation predict rapid decline in mild cognitive impairment. J Gerontol A Biol Sci Med Sci. 60(5):660-6.

[173] Liedorp M., van der Flier W.M., Hoogervorst E.L., Scheltens P., and Stam C.J., 2009. Associations between patterns of EEG abnormalities and diagnosis in a large memory clinic cohort. Dement Geriatr Cogn Disord. 27(1):18-23.

[174] Haupt M., Gonzlez-Hernndez J.A., and Scherbaum W.A., 2008. Regions with different evoked frequency band responses during early-stage visual processing distinguish mild Alzheimer dementia from mild cognitive impairment and normal aging. Neurosci Lett. 442(3):273-8.

[175] Roks G., Korf E.S., van der Flier W.M., Scheltens P., and Stam C.J., 2008. The use of EEG in the diagnosis of dementia with Lewy bodies. J Neurol Neurosurg Psychiatry. 79(4):377-80.

[176] Rosengarten B., Paulsen S., Molnar S., Kaschel R., Gallhofer B., and Kaps M., 2007. Activation-flow coupling differentiates between vascular and Alzheimer type of dementia. J Neurol Sci. 257(1-2):149-54.

[177] Kai T, Asai Y, Sakuma K, Koeda T, Nakashima K., 2005. Quantitative electroencephalogram analysis in dementia with Lewy bodies and Alzheimer's disease. J Neurol Sci. 237(1-2):89-95.

[178] Jeong J., Chae J.H., Kim S.Y., and Han S.H., 2001. Nonlinear dynamical analysis of the EEG in patients with Alzheimer's disease and vascular dementia. Journal of Clinical Neurophysiology 18(1): 58-67.

[179] Gawel M., Zalewska E., Szmidt-Sałkowska E., and Kowalski J., 2009. The value of quantitative EEG in differential diagnosis of Alzheimer's disease and subcortical vascular dementia. J Neurol Sci.

[180] Uhlhaas P.J., Pantel J., Lanfermann H., Prvulovic D., Haenschel C., Maurer K., Linden D.E., 2008. Visual perceptual organization deficits in Alzheimer's dementia. Dement Geriatr Cogn Disord. 25(5):465-75. 
[181] Andersson M., Hansson O., Minthon L., Rosén I., and Londos E., 2008. Electroencephalogram variability in dementia with lewy bodies, Alzheimer's disease and controls. Dement. Geriatr. Cogn. Disord. 26(3):284-90.

[182] Bonanni L., Thomas A., Tiraboschi P., Perfetti B., Varanese S., and Onofrj M. EEG comparisons in early Alzheimer's disease, dementia with Lewy bodies and Parkinson's disease with dementia patients with a 2-year follow-up. Brain. 131:690705.

[183] Misulis K. E. and Fakhoury T., 2001. Spehlmann's Evoked Potential Primer. Butterworth-Heinemann, Boston-Oxford.

[184] Brown, C.M., and Hagoort P., 1999. The cognitive neuroscience of language. In Colin M. Brown and Peter Hagoort. The Neurocognition of Language. New York: Oxford University Press., p. 6.

[185] Crowley K., Sullivan E.V., Adalsteinsson E., Pfefferbaum A., and Colrain I.M., 2005. Differentiating pathologic delta from healthy physiologic delta in patients with Alzheimer disease. Sleep 28(7):865-70.

[186] Fernandez R., Kavcic V., and Duffy C.J., 2007. Neurophysiologic analyses of lowand high-level visual processing in Alzheimer disease. Neurology 68(24):2066-76.

[187] Crowley K., Trinder J., and Colrain I.M., 2004. Evoked K-complex generation: the impact of sleep spindles and age. Clin Neurophysiol. 115(2):471-6.

[188] Caravaglios G., Costanzo E., Palermo F., and Muscoso E.G., 2008. Decreased amplitude of auditory event-related delta responses in Alzheimer's disease. Int. J. Psychophysiol. 2008 Oct;70(1):23-32.

[189] Papaliagkas V., Kimiskidis V., Tsolaki M., and Anogianakis G., 2008. Usefulness of event-related potentials in the assessment of mild cognitive impairment. BMC Neuroscience 9:107.

[190] Frodl T., Hampel H., Juckel G., Bürger K., Padberg F., Engel R.R., Möller H.J., and Hegerl U., 2002. Value of event-related P300 subcomponents in the clinical diagnosis of mild cognitive impairment and Alzheimer's Disease. Psychophysiology 39(2):175-81.

[191] Polich J. and Corey-Bloom J., 2005. Alzheimer's disease and P300: review and evaluation of task and modality. Curr Alzheimer Res. 2(5):515-25.

[192] Scherg M., 1992. Functional imaging and localization of electromagnetic brain activity. Brain Topography 5:103-11. More information on BESA can be found at http://www. besa.de/.

[193] van Deursen J.A., Vuurman E.F., Smits L.L., Verhey F.R., Riedel W.J., 2009. Response speed, contingent negative variation and P300 in Alzheimer's disease and MCI. Brain Cogn. 69(3):592-9.

[194] van Deursen J.A., Vuurman E.F., van Kranen-Mastenbroek V.H., Verhey F.R., Riedel W.J., 2009. 40-Hz steady state response in Alzheimer's disease and mild cognitive impairment. Neurobiol Aging.

[195] Karrasch M., Laine M., Rinne J.O., Rapinoja P., Sinervä E., and Krause C.M., 2006. Brain oscillatory responses to an auditory-verbal working memory task in mild cognitive impairment and Alzheimer's disease. Int J Psychophysiol. 59(2):168-78.

[196] Olichney J.M., Iragui V.J., Salmon D.P., Riggins B.R., Morris S.K., and Kutas M., 2006. Absent event-related potential (ERP) word repetition effects in mild Alzheimer's disease. Clin Neurophysiol. 117(6):1319-30. 
[197] Sotero R.C. and Trujillo-Barreto N.J., 2008. Biophysical model for integrating neuronal activity, EEG, fMRI and metabolism. Neuroimage 39(1):290-309.

[198] Moretti V.D., Pievani M., Fracassi C., Binetti G., Rosini S., Geroldi C., Zanetti O., Rossini P.M., Frisoni G.B., 2009. Increase of Theta/Gamma and Alpha3/Alpha2 Ratio is Associated with Amygdalo-Hippocampal Complex Atrophy. J Alzheimers Dis.

[199] Pogarell O., Teipel S.J., Juckel G., Gootjes L., Möller T., Bürger K., Leinsinger G., Möller H.J., Hegerl U., and Hampel H., 2005. EEG coherence reflects regional corpus callosum area in Alzheimer's disease. J Neurol Neurosurg Psychiatry 76(1):109-11.

[200] Babiloni C., Frisoni G.B., Pievani M., Vecchio F., Infarinato F., Geroldi C., Salinari S., Ferri R., Fracassi C., Eusebi F., Rossini P.M., 2008. White matter vascular lesions are related to parietal-to-frontal coupling of EEG rhythms in mild cognitive impairment. Hum Brain Mapp. 29(12):1355-67.

[201] Teipel S.J., Pogarell O., Meindl T., Dietrich O., Sydykova D., Hunklinger U., Georgii B., Mulert C., Reiser M.F., Möller H.J., Hampel H., 2008. Regional networks underlying interhemispheric connectivity: An EEG and DTI study in healthy ageing and amnestic mild cognitive impairment. Hum Brain Mapp 30(7):2098-2119.

[202] Julkunen P., Jauhiainen A.M., Westerén-Punnonen S., Pirinen E., Soininen H., Könönen M., Pääkkönen A., Määttä S., and Karhu J., 2008. Navigated TMS combined with EEG in mild cognitive impairment and Alzheimer's disease: a pilot study. J. Neuroscience Methods 172(2):270-6.

[203] Musha T., Asada T., Yamashita F., Kinoshita T., Chen Z., Matsuda H., Uno M., Shankle W.R., 2002. A new EEG method for estimating cortical neuronal impairment that is sensitive to early stage Alzheimer's disease. Clin. Neurophysiol. 113(7):1052-8.

[204] Bobkova N., Vorobyov V., Medvinskaya N., Aleksandrova I., Nesterova I., 2008. Interhemispheric EEG differences in olfactory bulbectomized rats with different cognitive abilities and brain beta-amyloid levels. Brain Res. 2008.

[205] Gianotti L.R., Künig G., Faber P.L., Lehmann D., Pascual-Marqui R.D., Kochi K., Schreiter-Gasser U., 2008. Rivastigmine effects on EEG spectra and threedimensional LORETA functional imaging in Alzheimer's disease. Psychopharmacology (Berl) 198(3):323-32.

[206] Yener G.G., Güntekin B., Oniz A., and Başar E., 2007. Increased frontal phase-locking of event-related theta oscillations in Alzheimer patients treated with cholinesterase inhibitors. Int. J. Psychophysiol. 64(1):46-52.

[207] Kogan E.A., Verchovsky R.G., Neufeld M.Y., Klimovitsky S.Sh., Treves T.A., and Korczyn A.D., 2007. Long-term tetrahydroaminoacridine treatment and quantitative EEG in Alzheimer's disease. J. Neural Transm. Suppl. (72):203-6.

[208] Moraes Wdos S., Poyares D.R., Guilleminault C., Ramos L.R., Bertolucci P.H., Tufik S., 2006. The effect of donepezil on sleep and REM sleep EEG in patients with Alzheimer disease: a double-blind placebo-controlled study. Sleep 29(2):199-205.

[209] Babiloni C., Bosco P., Ghidoni R., Del Percio C., Squitti R., Binetti G., Benussi L., Ferri R., Frisoni G., Lanuzza B., Cassetta E., Anello G., Gurzì M., Bartesaghi S., Lizio R., Tombini M., and Rossini PM, 2007. Homocysteine and electroencephalographic rhythms in Alzheimer disease: a multicentric study. Neuroscience 145(3):942-54. 